\title{
Stall Margin Improvement in a Centrifugal Compressor through Inducer Casing Treatment
}

\author{
V. V. N. K. Satish Koyyalamudi ${ }^{1}$ and Quamber H. Nagpurwala ${ }^{2}$ \\ ${ }^{1}$ MSR School of Advanced Studies, Bangalore 560058, India \\ ${ }^{2}$ Department of Automotive and Aeronautical Engineering, Faculty of Engineering and Technology, \\ M. S. Ramaiah University of Applied Sciences, Bangalore 560058, India
}

Correspondence should be addressed to Quamber H. Nagpurwala; qhn1947@gmail.com

Received 29 December 2015; Revised 7 March 2016; Accepted 16 March 2016

Academic Editor: Ryoichi Samuel Amano

\begin{abstract}
Copyright ( 2016 V. V. N. K. S. Koyyalamudi and Q. H. Nagpurwala. This is an open access article distributed under the Creative Commons Attribution License, which permits unrestricted use, distribution, and reproduction in any medium, provided the original work is properly cited.
\end{abstract}

\begin{abstract}
The increasing trend of high stage pressure ratio with increased aerodynamic loading has led to reduction in stable operating range of centrifugal compressors with stall and surge initiating at relatively higher mass flow rates. The casing treatment technique of stall control is found to be effective in axial compressors, but very limited research work is published on the application of this technique in centrifugal compressors. Present research was aimed to investigate the effect of casing treatment on the performance and stall margin of a high speed, 4:1 pressure ratio centrifugal compressor through numerical simulations using ANSYS CFX software. Three casing treatment configurations were developed and incorporated in the shroud over the inducer of the impeller. The predicted performance of baseline compressor (without casing treatment) was in good agreement with published experimental data. The compressor with different inducer casing treatment geometries showed varying levels of stall margin improvement, up to a maximum of $18 \%$. While the peak efficiency of the compressor with casing treatment dropped by $0.8 \%-1 \%$ compared to the baseline compressor, the choke mass flow rate was improved by $9.5 \%$, thus enhancing the total stable operating range. The inlet configuration of the casing treatment was found to play an important role in stall margin improvement.
\end{abstract}

\section{Introduction}

Centrifugal compressors are widely used in various industries like oil and gas, aviation, refrigeration, and turbochargers. They require fewer stages to achieve a given pressure ratio and have relatively wider operating range compared to the axial compressors. The demand for compact compression systems in the recent years has led to increased pressure ratio per stage with increased aerodynamic blade loading and reduced stable operating range. Hence, there is a need to improve the compressor stability/stall margin using either active or passive techniques. The active stall control techniques, like air jets and oscillating inlet guide vanes, require activation mechanisms adding weight to the machine and reducing the operational reliability. On the other hand, the passive techniques operate independently without any aid of external controls. The casing treatment is one of the passive stall control techniques, used effectively in axial compressors.
However, in most cases, an increase in compressor stall margin is accompanied by a reduction in compressor isentropic efficiency.

Casing treatment involves placing a porous casing around the compressor rotor tip, and in effect, it is a technique to control the casing or shroud boundary layer. Pioneering work on this technique was done by NASA [1-4] to evaluate the effect of various casing treatment geometries on stall margin and overall performance of axial compressors. Every conceivable configuration, ranging from simple honeycombs and circumferential grooves to axial and skewed slots, was tested, both under clean and distorted inlet flow conditions. Gains in stall margin up to $30 \%$ were reported. Also, the technique was found to be more effective for distorted flow than for uniform flow. Parametric studies carried out by Guruprasad et al. [5] on a transonic axial compressor stage showed that the axially and radially skewed treatment slots improved the stall margin by almost $25 \%$. Studies 
also revealed an optimum axial coverage of the treatment slots over the rotor tip. Experimental studies by Takata and Tsukuda [6] on a low speed compressor with skewed slot casing treatment configuration suggested that a dynamic flow mechanism involving inflow from the main stream into the slots within the stall cell and a jet-like flow discharging from the slots to the main flow outside the stall cell was responsible for the improvement in stall margin. Investigations by Smith and Cumptsy [7] on a low speed isolated rotor having axial skewed slot casing treatment also showed that the removal of high swirl, high loss fluid from the rotor trailing end and its reintroduction into the main flow near the rotor leading end was mainly responsible for the effectiveness of casing treatment. Recent experimental studies by Alone et al. [8-10] on the effect of axially bent casing treatment in a transonic axial compressor stage have shown that the treatment slot parameters like rotor tip axial coverage and porosity as well as the presence of a plenum behind the treatment slots significantly influence the compressor performance and its stalling characteristics.

The first ever attempt to study the fluid dynamic mechanism of flow through casing treatment was made by Boyce et al. [11]. Simulated studies on axial slots and circumferential grooves conducted in water (flow medium) using flow visualization technique led to the conclusion that the axial slots were superior to circumferential grooves. Takata and Tsukuda [6] used a 2D, linearized model of casing treatment to study the effect of fluid mass interchange between the treatment slots and the main flow. Another numerical study of the casing treatment with axial slots is reported by Crook et al. [12]. Two principal mechanisms of casing treatment effectiveness were identified, namely, suction of low total pressure, high blockage fluid at the rear of the passage, and energizing of the tip leakage flow, resulting in suppressing the blockage at its source. Numerical simulations by Wilke and Kau [13] have shown the influence of casing treatment slot location on the stability margin and efficiency of a transonic axial compressor stage. Unsteady numerical simulations by Shabbir and Adamczyk [14] have shown that the slots with different aspect ratios provide the same stability margin improvement for an axial flow rotor. Emmrich et al. [15] carried out unsteady flow simulations on a subsonic axial compressor stage with casing treatment comprising axially and radially skewed slots ending in a plenum chamber. It was reported that the flow separation over the rotor blade could be reduced by injecting recirculating flow with high radial velocity component at the location of high Mach numbers close to the suction side of the rotor blade.

There have been relatively few attempts to investigate the casing treatment technique in centrifugal compressors for improving the stall/surge margin. The first successful application of casing treatment technique in a centrifugal compressor was reported by Jansen et al. [16]. The treatment, comprising grooves/slots, was incorporated around the inducer region of the impeller. Experimental studies by Ding et al. [17] on a centrifugal compressor with holed casing treatment showed improvement in surge margin by $10 \%$ with negligible drop in efficiency. The occurrence of modal precursor waves was delayed by casing treatment.
Barton et al. [18] carried out numerical simulation on a vaned shroud incorporated in the inducer leading edge region of a centrifugal compressor impeller. The results confirmed the ability of the vaned shroud to extend the surge margin of the centrifugal compressor at part-speed operating conditions, while maintaining acceptable performance at design speed. Gao et al. [19] performed time accurate 3D numerical simulations of different casing treatment geometries on a high speed centrifugal compressor. The major mechanism of stall margin improvement was found to be the breakdown of the tip leakage vortex.

It may be noted that the inducer in a centrifugal compressor impeller behaves like an axial compressor rotor. It is, therefore, hypothesized that casing treatment with slotted casing/shroud around the inducer tip region may also be effective in controlling the suction surface and shroud boundary layers at low mass flow rate, thus delaying flow separation and consequently delaying the onset of rotating stall, with resulting improvement in stall margin. In the present studies, a high speed centrifugal compressor impeller has been numerically investigated with three configurations of slotted casing treatment at the inducer shroud. The streamwise shape of the treatment slots was designed to match with the aerofoil contour of inducer tip section. The treatment slot geometry was varied in terms of different upstream lengths and inlet configurations. The simulation results are presented in the form of global compressor performance and detailed flow behavior through impeller blade passages and casing treatment slots.

\section{Details of Centrifugal Compressor and Casing Treatment Configurations}

2.1. Baseline Centrifugal Impeller. A back-swept centrifugal impeller [20] of $4: 1$ total pressure ratio was considered for the present investigations. The impeller geometry and experimental performance data at $50 \%$ design speed are reported in [20]. Hence, the validation of the CFD simulations could be done only at $50 \%$ design speed. Subsequently the predicted performance of the impeller at $100 \%$ design speed was taken as the baseline performance for the casing treatment studies. Design specifications of the baseline impeller and its geometric details are given in Table 1 , and the CAD model of the impeller is shown in Figure 1. The present investigations were limited to centrifugal impeller only without any downstream diffuser and volute casing. The impeller tip clearance of $0.5 \mathrm{~mm}$ was uniform from inlet to exit.

2.2. Casing Treatment Configurations. The geometrical details of the three casing treatment (CT) configurations in meridional plane are shown in Figure 2. The CT configurations comprised slots of $20 \mathrm{~mm}$ depth, equal to $25 \%$ inducer leading edge height, designed to fully cover the inducer tip and also to extend upstream of the inducer leading edge. It was expected that the incoming low momentum fluid near the casing (shroud) will be partially sucked into the slots and subsequently mixed with the main flow at inducer exit. Hence, the shroud boundary layer at 


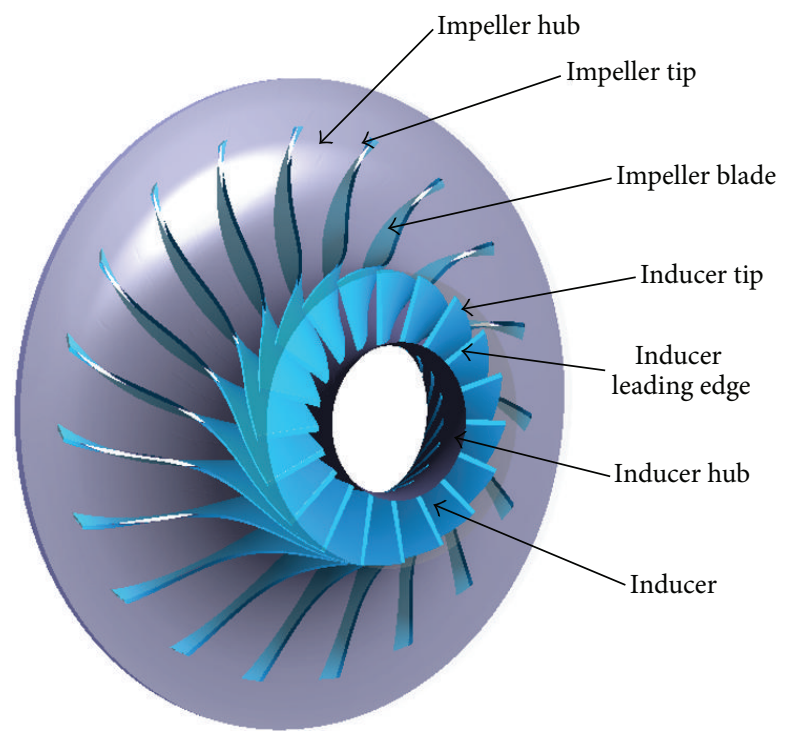

FIGURE 1: CAD model of centrifugal compressor impeller with shroud removed.

TABLE 1: Design specification of centrifugal impeller.

\begin{tabular}{lc}
\hline Parameter & Value \\
\hline Total pressure ratio & $4: 1$ \\
Rotational speed & $30,000 \mathrm{rpm}$ \\
Corrected mass flow rate & $2 \mathrm{~kg} / \mathrm{s}$ \\
Isentropic efficiency & $90 \%$ \\
Number of impeller blades & 19 \\
Inducer tip diameter & $160 \mathrm{~mm}$ \\
Inducer hub diameter & $80 \mathrm{~mm}$ \\
Impeller tip diameter & $300 \mathrm{~mm}$ \\
Impeller blade width at tip & $6.94 \mathrm{~mm}$ \\
Impeller blade tip clearance & $0.5 \mathrm{~mm}$ \\
Impeller back sweep angle & 30 degrees (from radial direction) \\
\hline
\end{tabular}

inducer exit will be relatively thinner with reduced chance of separation in the convex region of the main impeller. The three CT configurations, designated as CT-1, CT-2, and CT-3, differed in their inlet shape and length of the upstream extension. The geometric details of these three CT configurations are listed in Table 2.

The motivation for the present study was derived from Barton et al. [18], wherein an axial vaned shroud was investigated in a centrifugal compressor. However, the casing treatment vanes (slots) in the present study have the meridional shape matching with the aerofoil shape at inducer tip, and this shape is maintained over their entire radial height from inducer tip. A slightly enlarged view of the impellers with CT-1 and CT-2 casing treatment configurations is shown in Figures 3 and 4 for clarity. The third configuration, namely, CT-3, is similar to CT-2 with difference only in the upstream length of the treatment slots. Hence, the description below is better understood by referring to Figures $2-4$. The total length of casing treatment slots (vanes) comprises a rear part equal to $30 \%$ blade tip chord downstream of the inducer leading edge and a forward part equal to $50 \%$ inducer leading edge height for CT-1 and CT-2 and 100\% inducer leading edge height for CT-3 upstream of the inducer leading edge. The treatment slots extend radially into the inducer casing, and the treatment slot height is equal to $25 \%$ inducer leading edge height. The leading and trailing ends of these casing treatment slots join with the casing or hub (Figures 2 and 3), as the case maybe, with curves of $15 \mathrm{~mm}$ radius, thus avoiding sharp corners at the junctions. While the compressor inlet diameter for CT-1 is equal to the outer diameter of the treatment slots (Figure 2), the same is equal to the inducer tip diameter in case of CT-2 (and CT-3). Thus, although the compressor inlet diameter is larger than the inducer tip diameter for CT-1, the flow will encounter the same area at inducer leading edge as for impellers with CT-2 and CT-3. It may also be noted that the extension of casing treatment slots upstream of the inducer leading edge is done by maintaining their angle at the same value as the inducer tip leading edge angle of the impeller.

It was hypothesized that the shape of treatment slots matching with the inducer tip profile will have better flow recirculation dynamics leading to improvement in compressor performance, especially the stability margin. With this concept in mind, the three variants of CT were chosen to investigate the effect of the geometric changes. It may be noted that, unlike casing treatment studies in axial compressors, the application of casing treatment in centrifugal compressors is not reported much in open literature. The present investigations may be regarded as leading steps in this area to provide motivation for further parametric studies.

The CAD models of the baseline centrifugal impeller (Figure 1) and those with casing treatment configurations (Figures 3 and 4) were generated using UNIGRAPHICS-NX software. Since the impeller model was cyclic symmetric, only one blade passage was considered for CFD analysis with 
TABLE 2: Geometry details of casing treatment configurations.

\begin{tabular}{lll}
\hline S. number & Parameter & Geometric details \\
\hline 1 & Number of slots & 76 (4 slots per blade passage) \\
2 & Slot thickness & $3.9 \mathrm{~mm}$ \\
3 & Gap between two adjacent slots & $2.7 \mathrm{~mm}$ \\
4 & Slot profile in radial direction & Linearly extended in spanwise direction of impeller blade \\
5 & Slot radial depth & $20 \mathrm{~mm}$ (25\% of leading edge blade height) \\
& & Following the impeller blade angle distribution at inducer tip section from \\
6 & Slot profile in streamwise direction & leading edge \\
& & Extending linearly in upstream direction from leading edge of inducer at an \\
& Slot extension downstream of the inducer & angle to the inducer leading edge angle \\
7 & leading edge (over impeller blade tip) & $30 \%$ of impeller blade chord at shroud \\
& & CT- $1: 20 \mathrm{~mm}$ \\
& Slot extension upstream of inducer leading edge & CT-2: $20 \mathrm{~mm}$ Note: CT-1 and CT-2 differ in slot leading edge geometry \\
& & CT-3: $40 \mathrm{~mm}$ \\
\hline
\end{tabular}

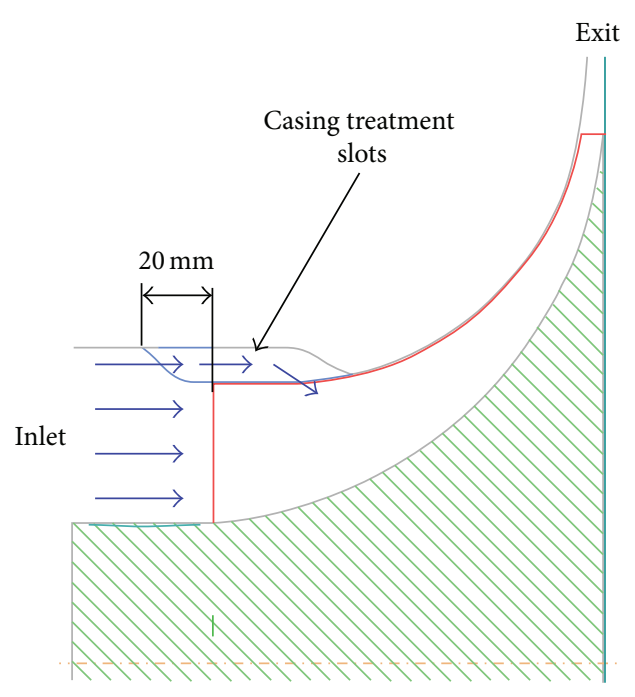

(a) CT-1

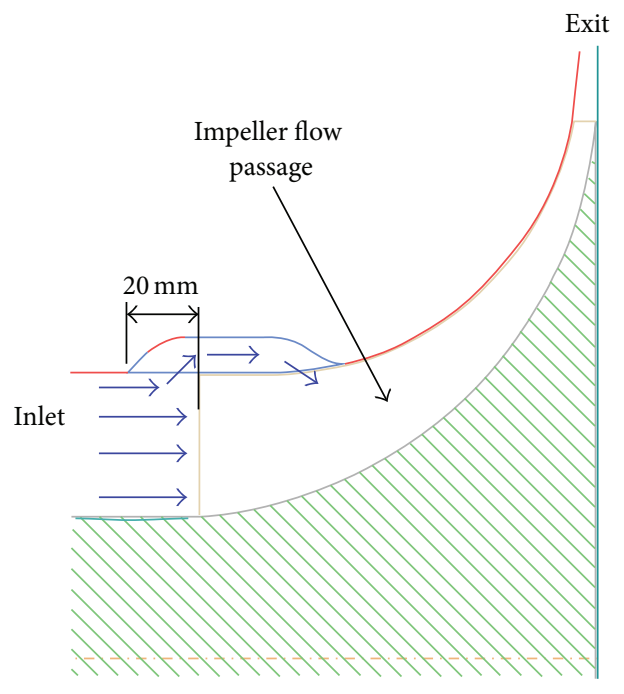

(b) CT-2

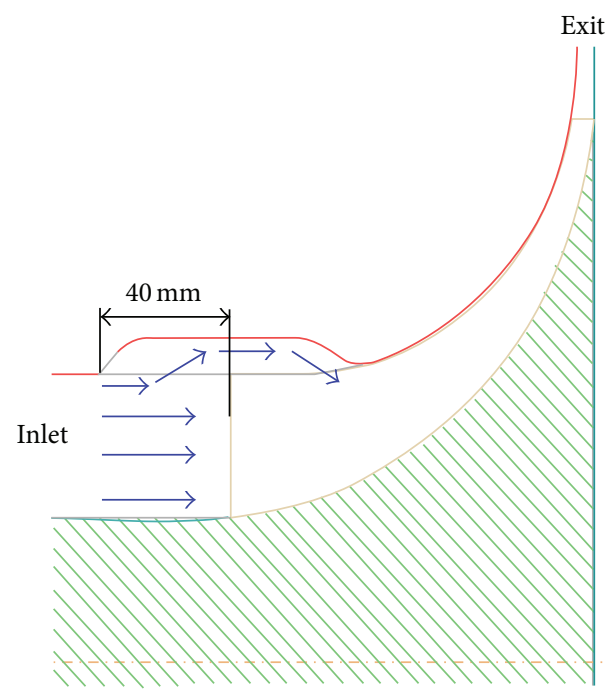

(c) CT-3

FIGURE 2: Casing treatment configurations in meridional plane. 


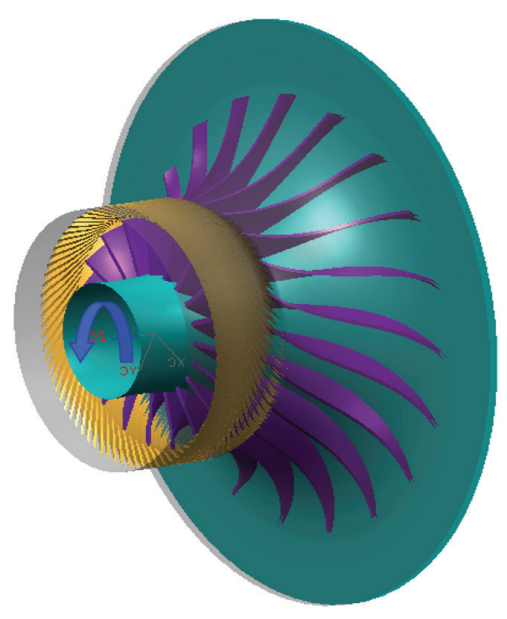

(a) Impeller with CT-1

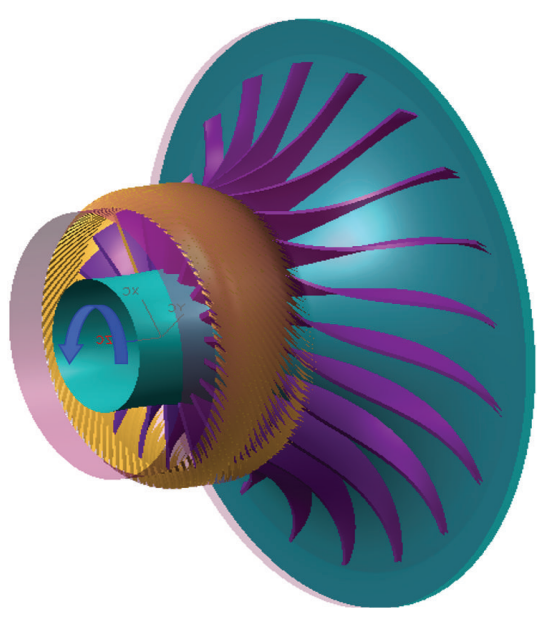

(b) Impeller with CT-2

FIGURE 3: CAD models of impellers with CT-1 and CT-2 highlighting the difference in shape of casing around the inducer.

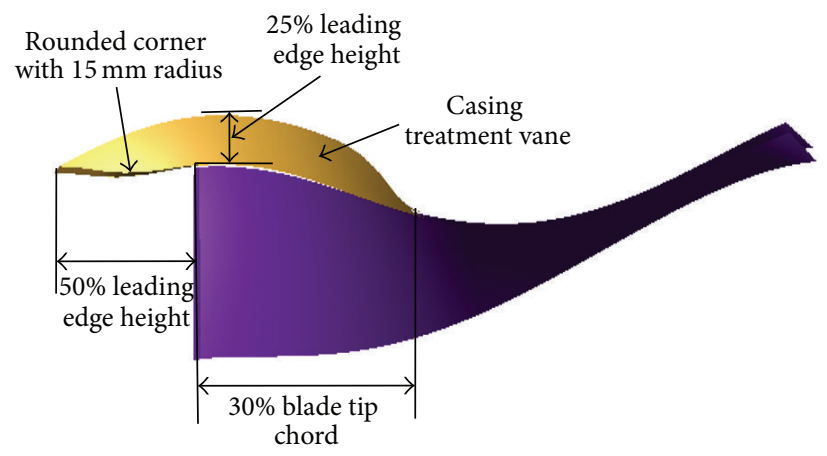

(a) Impeller with CT-1

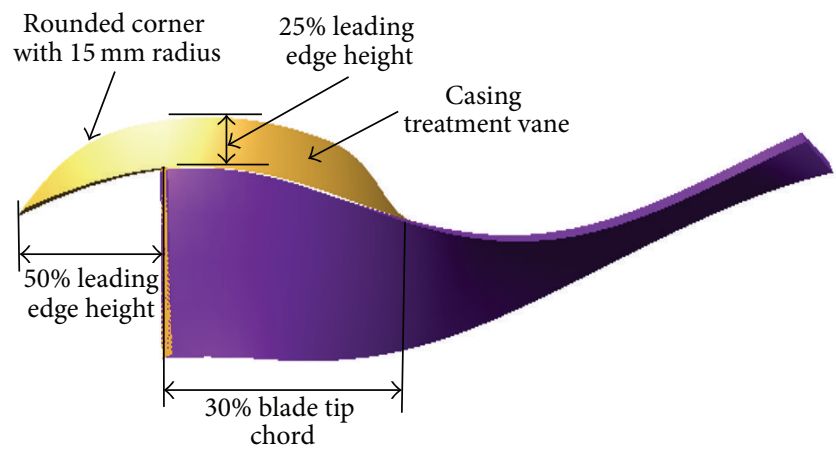

(b) Impeller with CT-2

FIGURE 4: CAD models of single impeller blade with CT-1 and CT-2, highlighting the position and proportions of casing treatment vane/slot. (For impeller with CT-3, the upstream length is $100 \%$ of leading edge height.)

proper periodic boundary conditions to represent the full impeller. This consideration facilitated a significant reduction in modeling, fluid domain discretization, and computation time.

\section{Fluid Domain Discretization and Solver Settings}

Figures 5 and 6 show the periodic computational domains of the baseline impeller and of the impeller with casing treatment CT-1, respectively, extracted using UNIGRAPHICS-NX software. The baseline impeller domain (Figure 5) comprises one blade passage whereas the impeller domain with casing treatment (Figure 6) comprises one blade passage and four casing treatment slots. The fluid domain was extended upstream by twice the inducer height and downstream by six times the impeller exit width to ensure flow stability and uniformity at domain inlet and exit. Design tip clearance between impeller and shroud was $0.5 \mathrm{~mm}$, uniform from inlet to exit. The computational domains for impeller with CT-2 and CT-3 were similar to that for impeller with CT-1.

Two computational fluid domains were created: (1) a rotating domain for impeller passage and (2) a stationary domain for casing treatment slots. These domains were discretized with structured grids having hexahedral elements using ICEM CFD software and multiblocking technique. The H-O-H mesh topology was employed for impeller flow domain. The O-grid topology was employed around the impeller to capture flow behavior accurately. Typical grids for impeller alone and the casing treatment slots are shown in Figures 7 and 8, respectively. Grid density was kept sufficiently large in the impeller tip clearance region and within the casing treatment slots. A total of 13 elements were created between the interface plane and the tip of the slots to capture the fluid flow interaction between impeller blades and casing treatment slots. A fine grid was also generated near the wall regions of the slots to capture detailed flow structure. All the grid quality parameters in the casing treatment flow channels were maintained the same as that in the impeller 


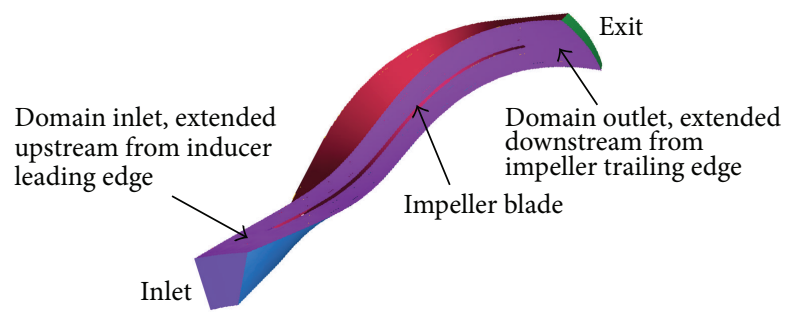

FIGURE 5: Computational domain of baseline impeller with one blade passage and inlet and exit domain extensions.

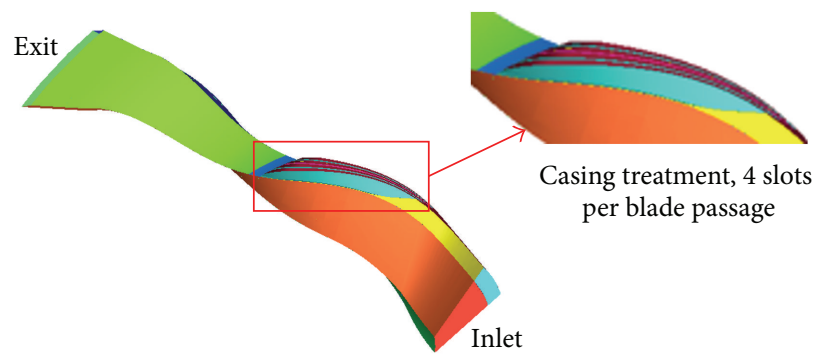

FIGURE 6: Computational domain of impeller with casing treatment CT-1 showing one blade passage and four treatment slots along with inlet and exit domain extensions.
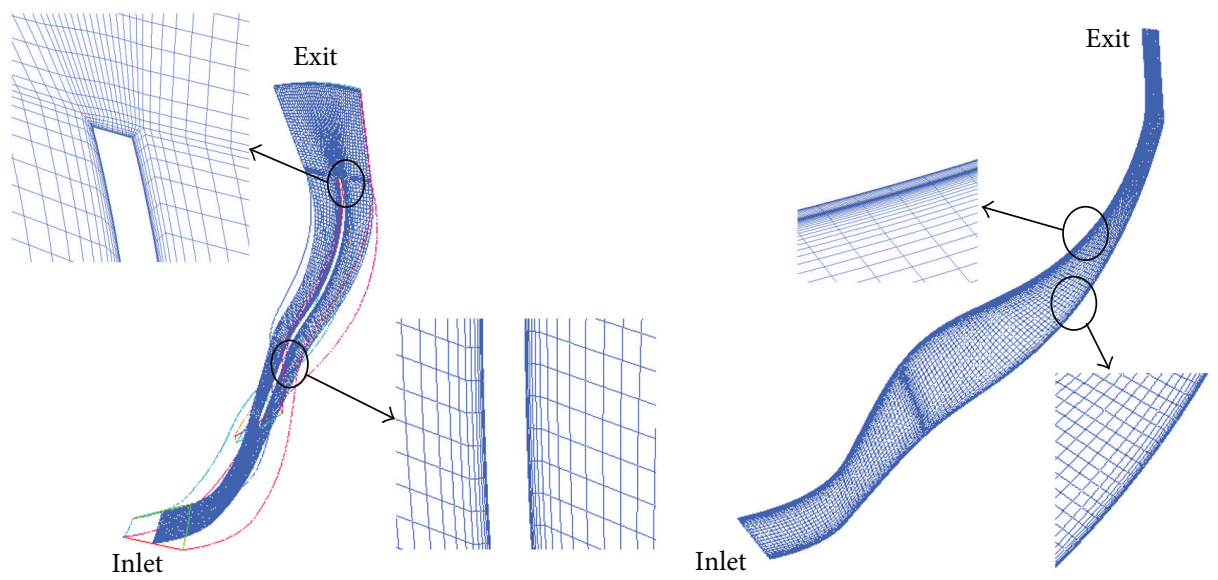

FIGURE 7: Computational grid for baseline impeller.

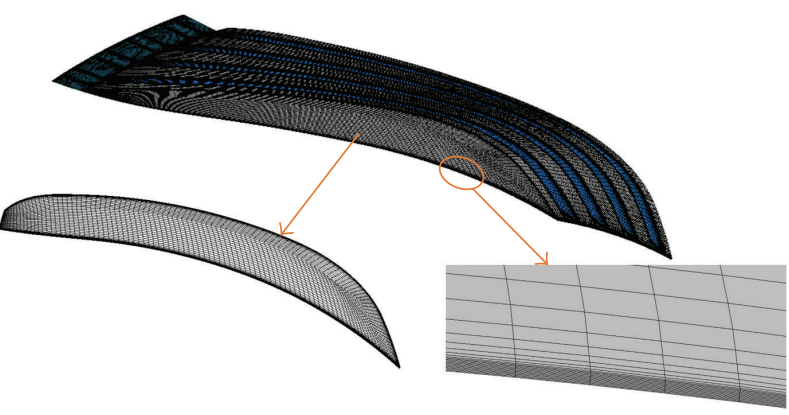

FIgURE 8: Computational grid for casing treatment slots. 


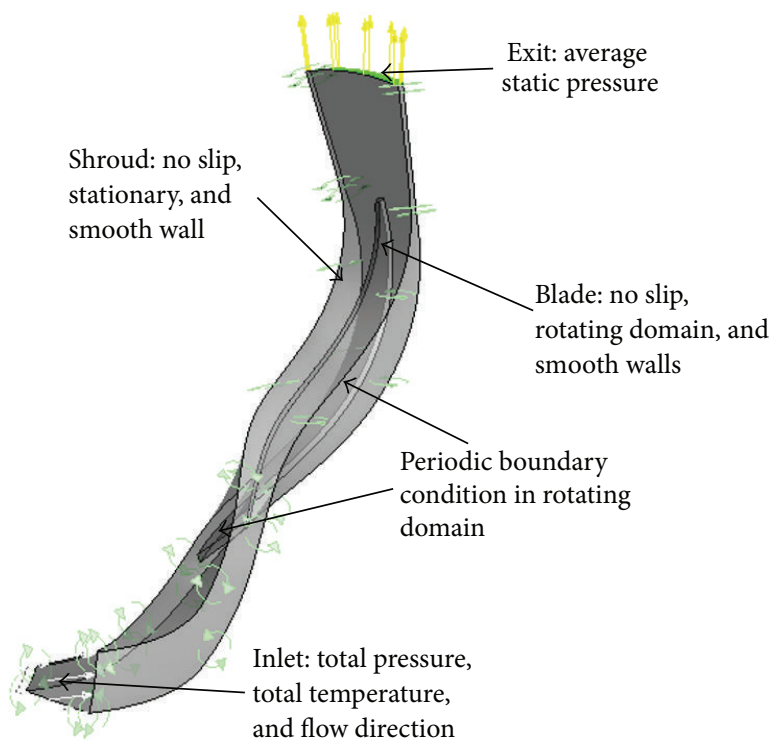

FIGURE 9: Boundary conditions applied to the impeller.

blade passage. Fine grid was also generated near the walls comprising impeller blade, hub, and shroud (Figure 7) to capture the boundary layer behavior on these surfaces. Impeller hub and blades were defined as rotating walls with no slip boundary condition. Shroud and casing treatment slots were defined as nonrotating walls with no slip. Rotational periodic conditions were applied at periodic planes of the impeller passage. A computational grid of good quality was ensured by keeping the elements with minimum face angle of $12^{\circ}$ and determinant greater than 0.35 . The maximum element volume ratio and edge length ratio had slightly higher values due to the boundary layer resolution $\left(y^{+}\right)$and restriction on maximum grid numbers. The values chosen were in line with those generally allowed for CFD computations. The first element height was generally maintained at $0.02 \mathrm{~mm}$ to get $y^{+}$of less than 30. A grid expansion ratio of 1.3 was used. The first element height was kept constant for all the grids used for grid independence study to avoid the influence of $y^{+}$. Figure 9 shows the boundary conditions applied to the impeller without and with casing treatment.

Steady state CFD simulations on the baseline impeller and the impellers with inducer casing treatment were carried out using ANSYS CFX software. The discretized form of Reynolds Averaged Navier-Stokes (RANS) equations was iteratively solved for all cases of baseline and treated impellers. The turbulence model used was SST $k-\omega$, because it is widely accepted that this model accurately predicts the near-wall flows with good resolution of boundary layer, flow separation, and so forth compared to $k$-e or standard $k-\omega$ turbulence models. It is recognized that while using SST $k-\omega$ turbulence model, the value of $y^{+}$should be low (single digit) for better resolution of the end wall, tip leakage, and boundary layer flows. However, a low $y^{+}$value demands very fine grid with consequent increase in grid density and computation time. With limited computational resources available with the authors, a nominal $y^{+}$value less than 30 was considered to be quite sufficient to establish the effectiveness of casing treatment in a centrifugal compressor with sufficient insight into the flow physics. In the sensitive regions, the $y^{+}$value was indeed closer to 10 .

The stage interface (mixing plane interface) was used between impeller and casing treatment domains. As the impeller rotates, the circumferential position of the impeller blades changes with respect to casing treatment slots; hence, stage interface is a more appropriate condition in this situation. The interface was created at the middle of the impellershroud tip gap.

All simulations, except the one for experimental validation, were carried out at $100 \%$ design speed. Air as ideal gas and with standard properties was used as working fluid in the compressor. The total pressure and total temperature, both at sea level conditions, were prescribed at domain inlet, while a variable static pressure was specified at domain exit. The flow was treated as compressible and it entered the impeller axially. To obtain the compressor performance characteristic from choke to stall mass flow rate, the exit static pressure (back pressure) was varied in discrete steps, allowing the solution to converge at each back pressure. All simulations were converged to a RMS residual value of $10^{-5}$. The numerical stall point was identified when, at a certain back pressure, the solution diverged. The stall point was resolved with a back pressure differential of $50 \mathrm{~Pa}$ between two consecutive values. The criterion for fixing the occurrence of numerical stall is quite well established. As the back pressure (static) is gradually increased from a low value in the choke region to higher values, the compressor performance moves towards design point and then towards stall. At some high back pressure, the CFD simulation does not converge and the solution diverges. This is termed as numerical stall. In the present studies, the numerical stall point was resolved with a difference in exit static pressure value of $50 \mathrm{~Pa}$ between last converged solution and the following diverged solution. 


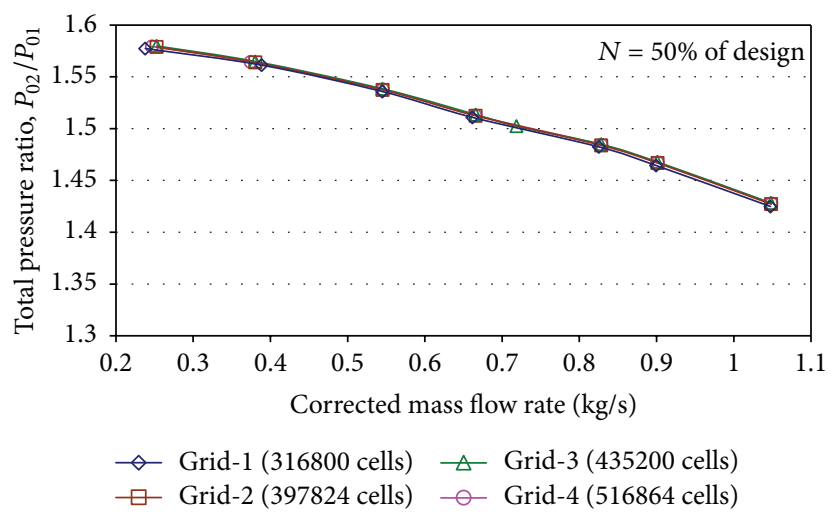

FIGURE 10: Grid independence study.

All the flow parameters were mass averaged at inlet and exit. The compressor total pressure ratio and adiabatic efficiency were calculated using the following equations:

$$
\begin{aligned}
& \text { Total pressure ratio }=\frac{P_{02}}{P_{01}}, \\
& \text { Isentropic efficiency }=\frac{\left(P_{02} / P_{01}\right)^{(\gamma-1) / \gamma}-1}{T_{02} / T_{01}-1} .
\end{aligned}
$$

Also, the corrected mass flow rate and corrected impeller rotational speed are defined as

$$
\begin{aligned}
& \text { Corrected mass flow rate }=\frac{\dot{m} \sqrt{\theta}}{\delta}, \\
& \text { Corrected rotational speed }=\frac{N}{\sqrt{\theta}} .
\end{aligned}
$$

\section{Results and Discussion}

The results of CFD simulations on the compressor without and with casing treatment are presented and discussed in this section. Initially, the grid independence and validation studies were carried out to select an appropriate grid density and also to confirm the accuracy level of the predicted data. Subsequently, the effects of casing treatment on compressor overall performance and on the flow development through the impeller blade passages are discussed.

4.1. Grid Independence and Validation Studies. Grid size plays an important role in both convergence and accuracy of the solution. Hence, a grid independence study was performed at 50\% design speed to ensure that the numerical solutions were grid independent. CFD simulations on the baseline impeller were performed with different grid sizes, having 316800 elements (Grid-1), 397824 elements (Grid-2), 435200 elements (Grid-3), and 516864 elements (Grid-4), using ANSYS CFX software. The first element height was kept constant at $0.02 \mathrm{~mm}$ for all the grid sizes to avoid the influence of $y^{+}$on the results. Figure 10 shows the variation of total pressure ratio with corrected mass flow rate at $50 \%$ design speed for the four different grid sizes. The compressor performance obtained from all these four grids is nearly the same. The ratio between the fine grid and the coarse grid is almost 1.63. It is unlikely that the predicted compressor performance will change with further increase in grid size. The difference between the results obtained with Grid-3 and Grid-4 is negligible. Hence, Grid-3, with 435200 elements, was used for subsequent simulations.

The experimental results for baseline compressor impeller were available at $50 \%$ design speed. Hence, the impeller performance from CFD simulation is validated at this speed only. The impeller performance was then computed at $100 \%$ design speed and used as the baseline performance for casing treatment studies. Figure 11 shows the experimental and predicted performance of the compressor without casing treatment at $50 \%$ design speed in terms of variation of total pressure ratio and isentropic efficiency with corrected mass flow rate. Considering the uncertainties in experiments as well as in numerical simulations, the agreement between the two data sets is fairly good. Referring to Figure 11, while the isentropic efficiency is predicted quite well, the pressure ratio is only slightly overpredicted (by $\max 2 \%)$ compared to the experimental values. However, the slopes of the performance curves are well reproduced. It is also to be noted that the test compressor exhibits a performance characteristic with negative slope only from choke mass flow rate to stall mass flow rate. A predicted peak pressure ratio of 1.58 occurs at stall mass flow rate of $0.23 \mathrm{~kg} / \mathrm{s}$. On the other hand, the isentropic efficiency increases with decrease in mass flow rate from choke point, reaches a maximum value of 0.93 at a mass flow rate of $1.1 \mathrm{~kg} / \mathrm{s}$, and then reduces with further reduction in mass flow rate up to the stall point. The predicted total range of mass flow rate seems significantly larger than the experimental mass flow range. One reason for this is that the experimental results, if any, were not available at higher mass flow rates. The experimental performance data was taken from the published literature. Another reason is that the experimental facility would have additional components like downstream diffuser and volute casing, whereas the numerical simulation is carried out only on the impeller. The presence of these downstream components (diffuser, volute casing, and exit 


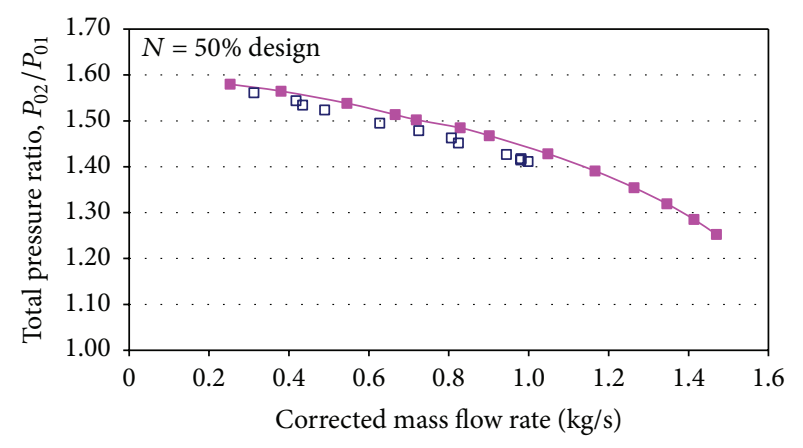

Experiment

$\rightarrow$ CFD

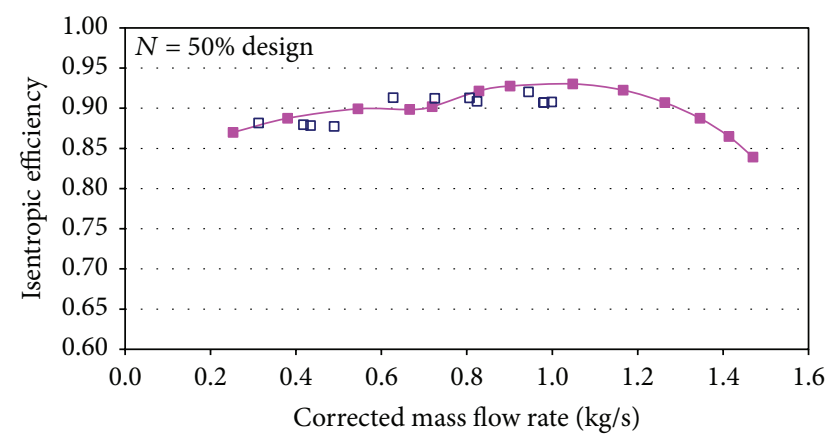

Experiment

- CFD

(a)

(b)

FIGURE 11: Validation study. Comparison of experimental and predicted performance of compressor without casing treatment at $50 \%$ design speed.

duct) would act as additional throttle, resulting in the compressor producing a lower mass flow rate than design and predicted values. The difference in experimental and predicted maximum mass flow rates is almost $0.5 \mathrm{~kg} / \mathrm{s}$ at $50 \%$ design rotational speed. Further, the experimental total pressure ratio was based on pressure measurements downstream of the volute (in the exit duct). In the higher mass flow range, the predicted compressor pressure ratio and efficiency agree quite well with the experimental data. Apparently, the experimental pressure ratio is slightly lower than the predicted one owing to losses in diffuser and volute. In view of the above, the validation of predicted compressor performance with the available experimental data is quite satisfactory.

\subsection{Compressor Performance without and with Casing Treat-} ment at Design Speed. Overall performance characteristics of the compressor at $100 \%$ design speed without and with three casing treatment configurations, namely, CT-1, CT-2, and CT-3, are shown in Figure 12. The total pressure ratio and isentropic efficiency are plotted against corrected mass flow rate from choke to stall point. Similar to $50 \%$ design speed, the total pressure ratio (Figure 12(b)) continuously increases from choke to stall as the mass flow rate is reduced (increase in back pressure). The peak pressure ratios for all impeller builds occur at the respective stall mass flow rates. For the baseline impeller, the predicted peak pressure ratio occurs at a mass flow rate of $\sim 1.4 \mathrm{~kg} / \mathrm{s}$. There is only small variation in the pressure ratio characteristic of the impellers with three casing treatment geometries. However, there is marked difference in the choke mass flow rate of baseline compressor and those with casing treatment. Incorporation of casing treatment slots over the inducer at the inlet increases the choke mass flow rate by almost $9.5 \%$ compared to the baseline compressor without CT. Thus the overall stable flow range with casing treatment is improved by inducer casing treatment. The casing treatment also results in reducing the stall mass flow rate, and the maximum gain of $18 \%$ in stall margin is obtained for the configuration CT-1. There is no stall margin improvement observed for casing treatment configuration CT-2, whereas for casing treatment configuration CT-3, a marginal stall margin improvement of $3.6 \%$ is achieved compared to the baseline impeller. The maximum total pressure ratio at stall point of CT-1 is 4.45 as compared to the maximum total pressure ratio of 4.3 at stall point of baseline compressor.

The isentropic efficiencies (Figure 12(a)) of all the impellers, without and with casing treatment, increase as the mass flow rate is reduced from the respective choke points, reach a maximum, and drop again with further reduction in mass flow rate till the respective stall points. A peak efficiency of $90 \%$ for baseline impeller occurs at a mass flow rate of $1.95 \mathrm{~kg} / \mathrm{s}$. However, slightly lower peak efficiencies of 89-89.5\% for the impellers with casing treatment occur at a higher mass flow rate of $\sim 2.2 \mathrm{~kg} / \mathrm{s}$. Further, the efficiency characteristics of impellers with casing treatment are flatter than that of the baseline impeller. Among the three casing treatment geometries, the characteristic for CT-1 is flatter than the other two with highest peak efficiency of $89.5 \%$ and also a higher efficiency of $88 \%$ at lowest stall mass flow rate of $1.15 \mathrm{~kg} / \mathrm{s}$. In fact, the impeller with CT-1 exhibits higher isentropic efficiency throughout the mass flow rate range beyond choke region. Thus, overall the casing treatment configuration CT-1 appears to be better among all the three CT configurations tested in improving the compressor performance.

4.3. Flow through Baseline Impeller Blade Passage. The variations of circumferentially averaged relative Mach number through the blade passage of the baseline impeller at neardesign and stall mass flow rate are shown in Figure 13.

At near-design mass flow rate (Figure 13(a)), there are low Mach number regions both at the shroud and at the hub walls downstream of the inducer. Further downstream, in the radial part of the impeller, the flow accelerates to higher Mach numbers that are eventually lower than the inlet relative Mach number, indicating overall flow deceleration through the impeller. Beyond the impeller tip, at domain exit, the relative Mach numbers are high owing to fluid rotation with increasing tangential velocity in the extended flow domain. At stall mass flow rate (Figure 13(b)) and under the influence 


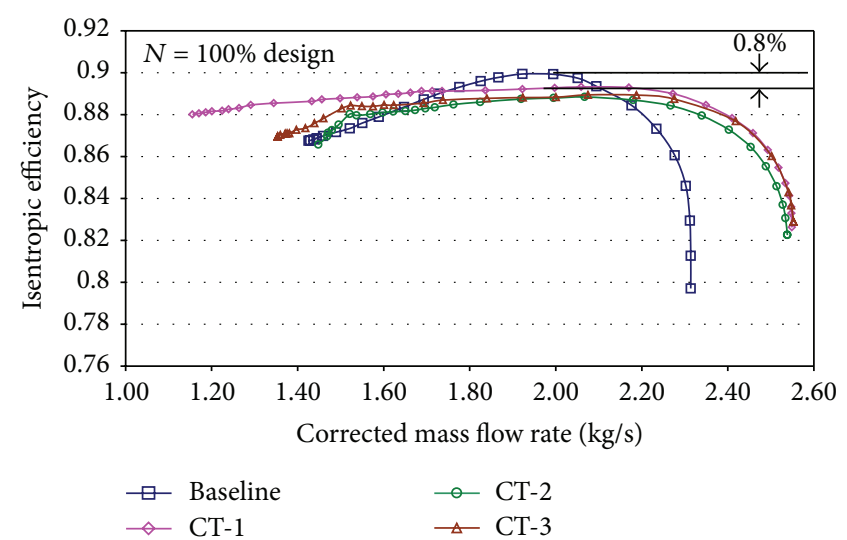

(a)

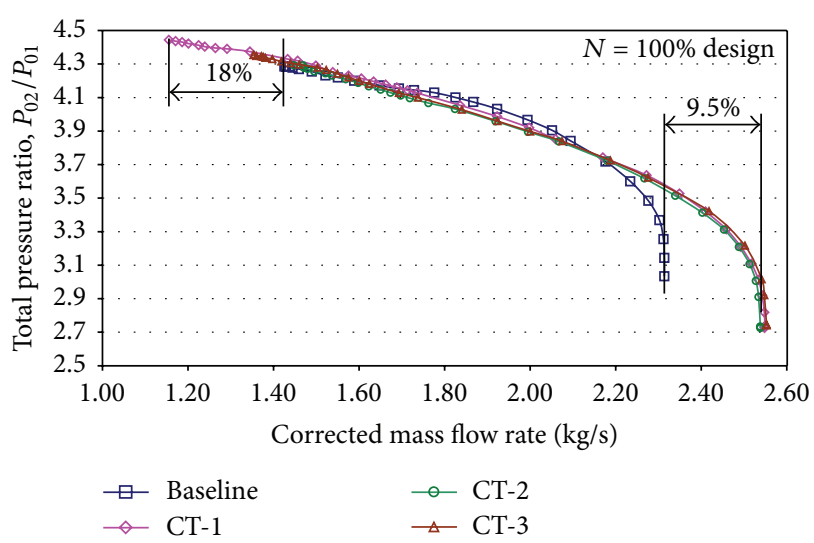

(b)

FIGURE 12: Overall performance characteristics of compressor without and with inducer casing treatment.

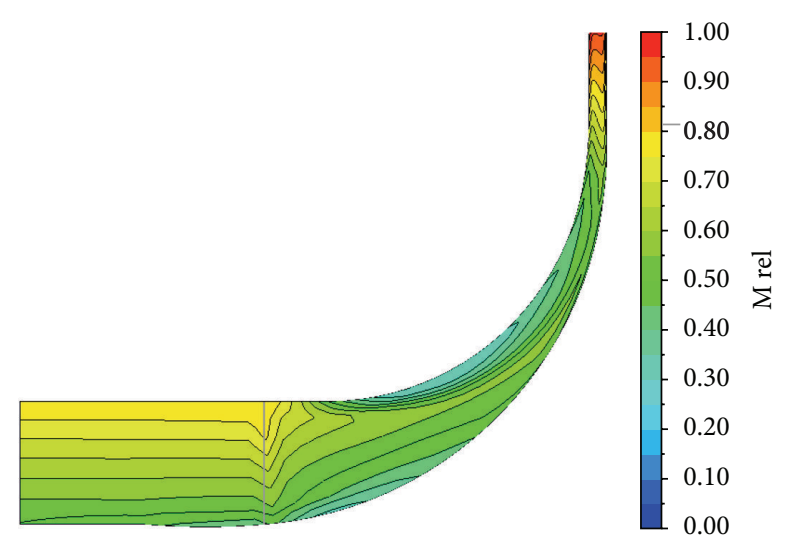

(a) Near-design mass flow rate

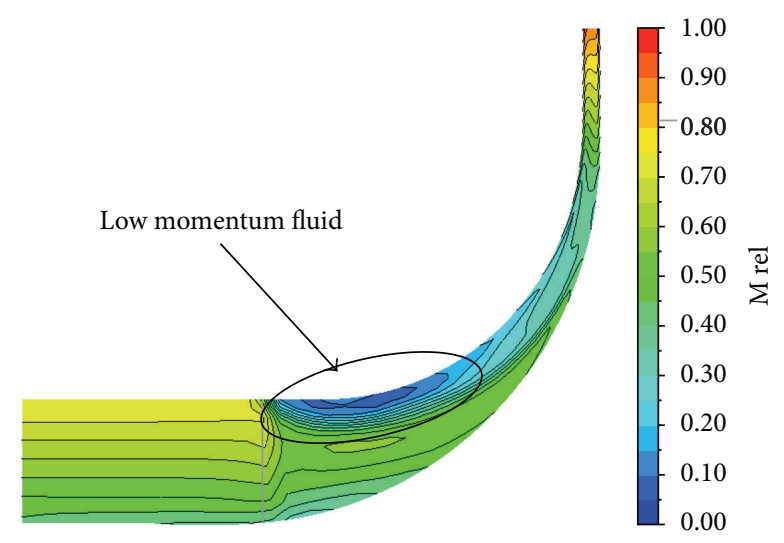

(b) Stall mass flow rate

FIGURE 13: Circumferentially mass averaged relative Mach number distribution in impeller blade passage.

of larger adverse pressure gradient, the low momentum recirculating fluid zone at the shroud is intensified as it moves upstream, occupying even some part of the inducer. However, as in case of near-design mass flow rate, the flow accelerates further downstream towards impeller exit. The relative Mach number at the domain exit is lower than that at near-design mass flow rate.

Figure 14 shows the distribution of static pressure on the suction and pressure surfaces of the impeller blade at three spanwise locations of $20 \%, 50 \%$, and $90 \%$ blade height from hub wall and at two mass flow rates corresponding to near-design and stall. At both the mass flow rates, the blade loading increases from inlet to exit of the impeller. At stall flow rate (Figure 14(b)), the blade loading near the shroud (90\% impeller blade height) is very low in the inducer region and downstream owing to flow separation on the blade suction surface at high positive incidence angles (see Figure 13(b) also). The static pressures on both suction and pressure surfaces are lower at $90 \%$ span compared to the values at $20 \%$ and $50 \%$ span. The flow blockage in the impeller blade passage at low mass flow rate leads to inception of stall.
4.4. Flow Development in Impeller Blade Passage with Casing Treatment. The relative effectiveness of different inducer casing treatment configurations CT-1, CT-2, and CT-3 can be better explained with the help of (i) absolute velocity vectors through the inducer flow passage (see observation plane in Figure 15) and casing treatment slots, as shown in Figure 16, and (ii) distribution of circumferentially mass averaged relative Mach number in the impeller blade passage in meridional plane, as shown in Figure 17, for the baseline and three treated impeller geometries. The impeller length covered in Figure 16 includes only the inducer and casing treatment slots. The compressor operating point corresponds to stall mass flow rate of the baseline impeller. For the baseline impeller (Figure 16(a)), a large recirculation zone between $70 \%$ and $100 \%$ blade span is observed, as seen in Figures 13(b) and 17(a) also. This low momentum, recirculating flow extends over $30 \%$ of the impeller blade length downstream of the inducer. The partial blockage produced by this low momentum fluid eventually leads to inception of stall.

In the presence of casing treatment CT-1 (Figures 16(b) and $17(\mathrm{~b}))$, the recirculation zone is significantly reduced 


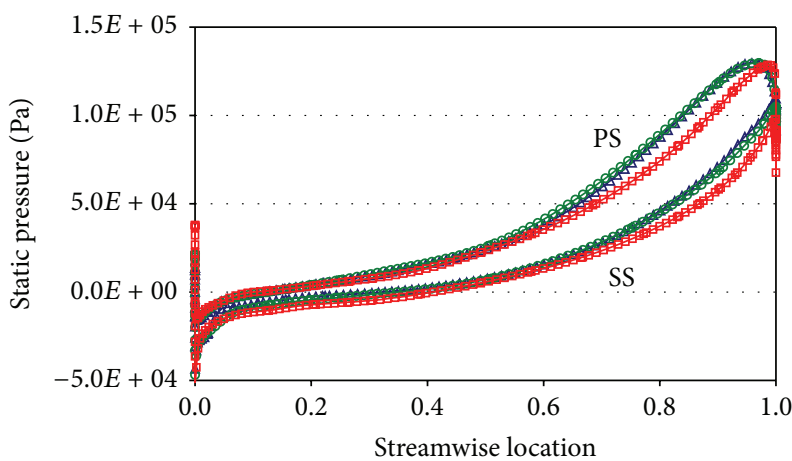

$\triangle$ Baseline, $20 \%$ span $\rightarrow-$ Baseline, $90 \%$ span - Baseline, $50 \%$ span

(a) Blade loading at near-design mass flow rate $(2 \mathrm{~kg} / \mathrm{s})$

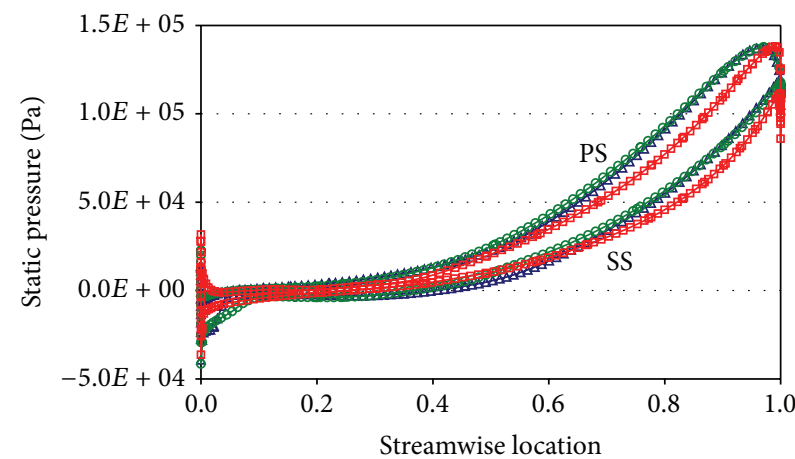

$\triangle$ Baseline, $20 \%$ span $\neg$ Baseline, $90 \%$ span - $\bigcirc$ Baseline, $50 \%$ span

(b) Blade loading at stall mass flow rate $(1.43 \mathrm{~kg} / \mathrm{s})$

FIGURE 14: Impeller blade loading at three spanwise locations of $20 \%, 50 \%$, and $90 \%$ from hub wall at near-design and stall mass flow rate.

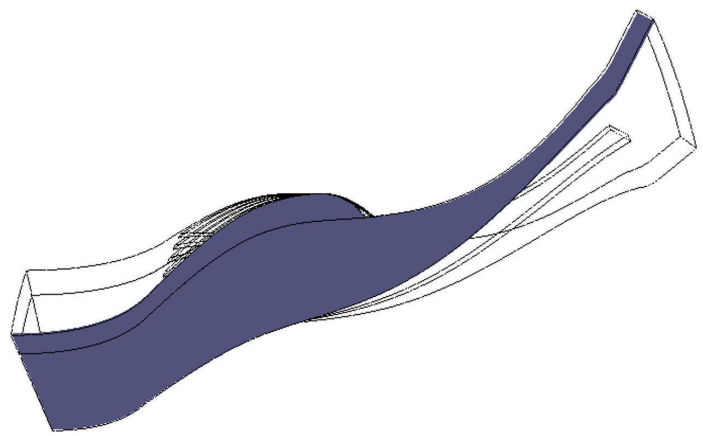

FIGURE 15: Location of periodic plane for plotting velocity vectors.

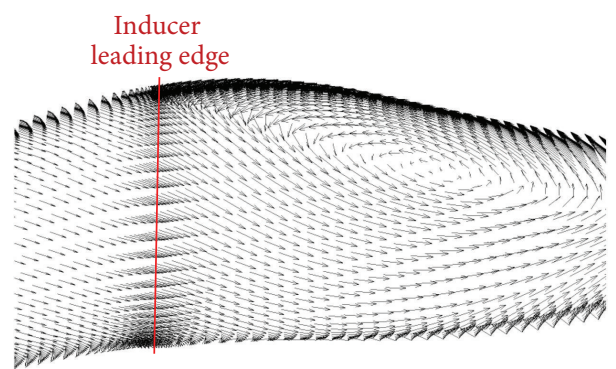

(a) Baseline impeller

Inducer

leading edge

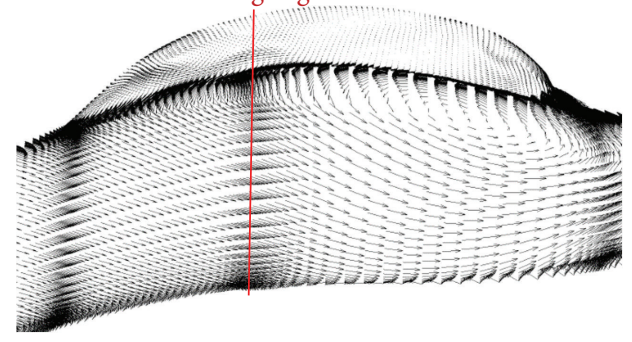

(c) Impeller with CT-2

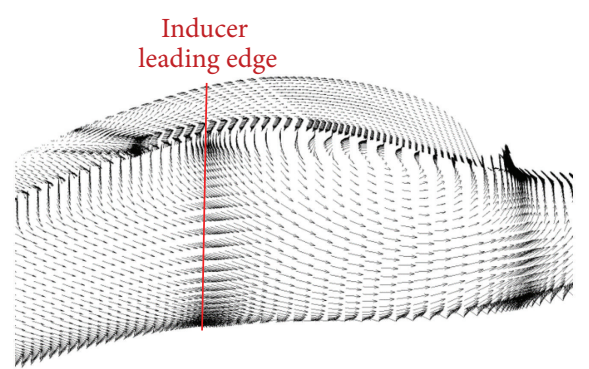

(b) Impeller with CT-1

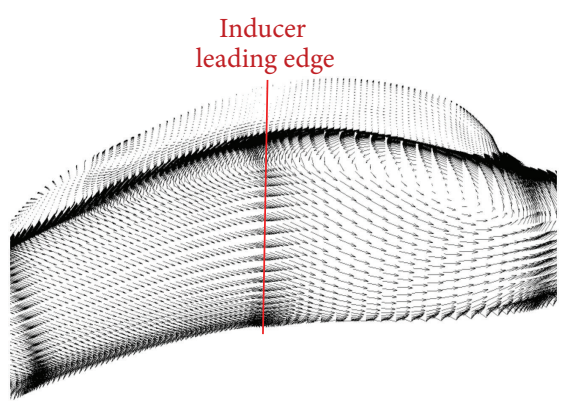

(d) Impeller with CT-3

FIGURE 16: Absolute velocity vectors in the inducer channel and in the casing treatment slots. 


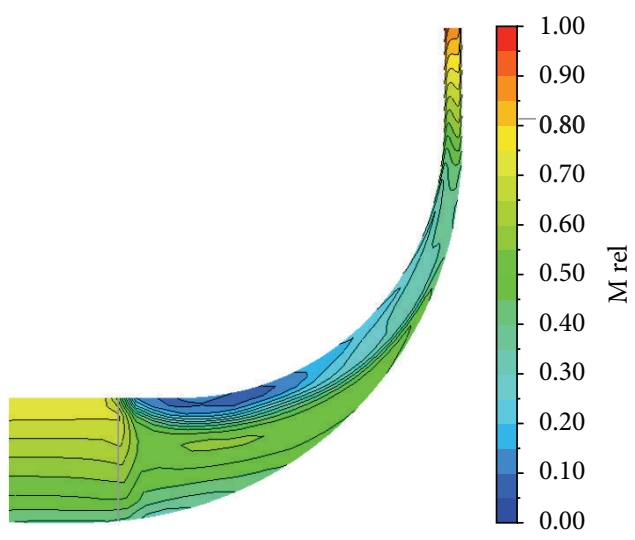

(a) Baseline impeller

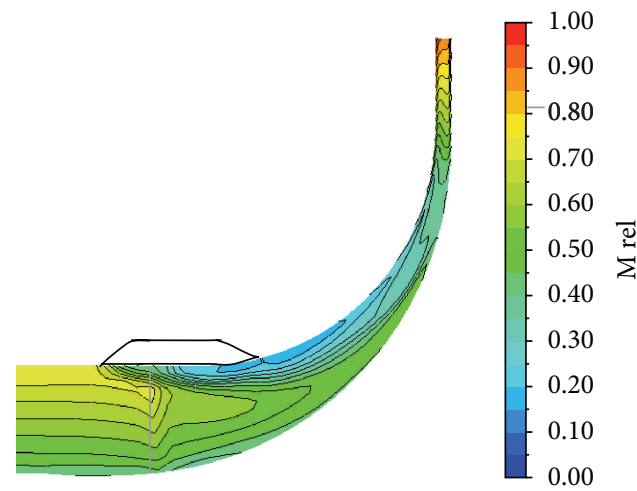

(c) Impeller with CT-2

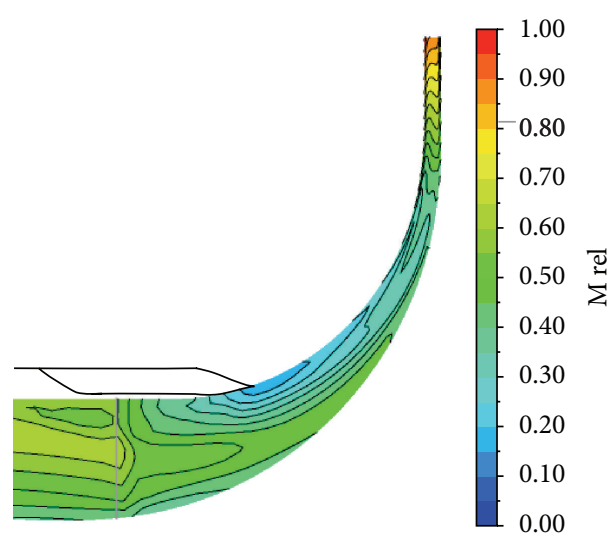

(b) Impeller with CT-1

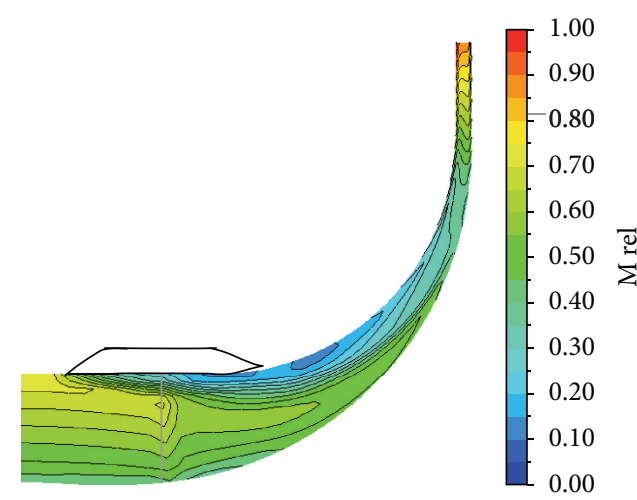

(d) Impeller with CT-3

FIGURE 17: Circumferentially mass averaged relative Mach number distribution in the meridional plane for impellers without and with three casing treatment geometries at stall mass flow rate of baseline compressor.

and is moved downstream. The energization of flow near the shroud compared to baseline impeller is evident (Figure 17(b)). It may be noted that in case of CT-1 the compressor inlet diameter is larger than the inducer tip diameter. Hence, the incoming flow enters straight into the treatment slots passages and moves forward (Figure 16(b)). Of course there will be leakage flow between the inducer tip and the treatment slots, but the momentum of the incoming flow entering straight into the slots continues to push the flow forward. This energizes the recirculation zone present in the baseline impeller and moves it downstream. Hence, the incidence angle at inducer inlet in the tip region increases, allowing the mass flow rate to be reduced beyond the stall mass flow rate of baseline impeller with plane shroud with consequent increase in stall margin, as shown in Figure 12. There is indication of localized reverse flow in the vicinity of the inducer tip, but, in general, the flow quality in the impeller passage is greatly improved compared to the baseline configuration.

In case of treatment configurations CT-2 and CT-3, the scenario is quite different (Figures 16(c) and 16(d)). The incoming flow mainly passes through the inducer blade passage with very low forward absolute velocities within the treatment slots. In fact, in the lower part of the slots, that is, near the inducer tip clearance, the flow is reversed (Figures 16(c) and 16(d)). This reverse flow region near the inducer tip extends throughout the treatment slot length.
The recirculation flow region near the shroud is stronger than for CT-1 impeller and extends upstream of leading ends of CT-2 and CT-3 up to a distance much beyond their respective trailing ends (Figures $17(\mathrm{c})$ and $17(\mathrm{~d})$ ). The effect of these strong reverse flows and large recirculating flows in the vicinity of the inducer tip compared to CT- 1 is to cause increased blockage and intolerance to positive incidence angle at lower mass flow rates, resulting in inception of stall at mass flow rates comparable with the baseline impeller. Unlike CT-1 impeller, the incoming flow entering the straight portion of the treatment slots in CT-2 and CT-3 impellers through an initial curved path has relatively lower momentum, resulting in relatively lower forward flow velocities through the slots. Figure 17 also shows that the flow field upstream of the inducer leading edge is considerably influenced by the presence of the casing treatment.

From the above discussion, it is evident that among the three casing treatment configurations, the CT- 1 configuration produces the largest gain in stall margin. Hence, the subsequent discussions are restricted to the comparison of flow behavior in baseline and CT-1 impellers.

\subsection{Effect of Casing Treatment on Impeller Blade Loading.} The variations of static pressure on the suction and pressure surfaces of the blades of baseline and CT- 1 impellers at 90\% span are shown in Figure 18. These blade loadings 


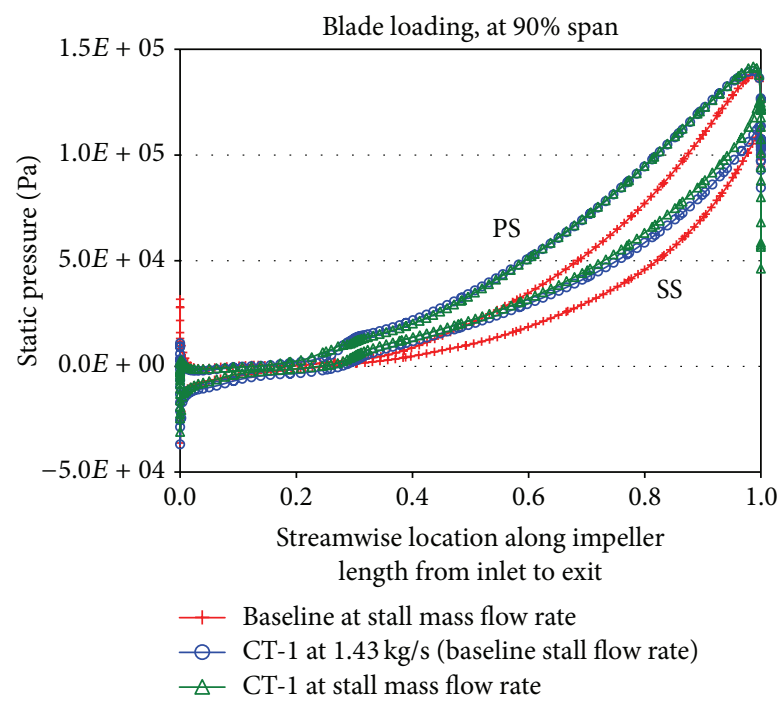

FIGURE 18: Comparison of blade loading of baseline impeller and impeller with CT-1 casing treatment at respective stall mass flow rates.

are presented for (1) baseline impeller at its own stall mass flow rate, (2) CT-1 impeller at the stall mass flow rate of baseline impeller, and (3) CT-1 impeller at its own stall mass flow rate. With the introduction of casing treatment (CT-1), the static pressure on both suction and pressure surfaces of the impeller improves compared to the baseline impeller at the stall mass flow rate of baseline impeller. Also, the flat region near $20 \%$ streamwise location is removed, indicating improvement in the recirculation flow region of baseline impeller. As the mass flow rate is further reduced to the stall point of CT-1, there is no appreciable change in the static pressure distribution compared to the one at higher mass flow rate corresponding to the stall mass flow rate of baseline impeller. However, there is substantial rise in total pressure (Figure 11(a)) between the two operating points, indicating that there is more contribution from increase in dynamic pressure rise than static pressure rise.

4.6. Flow Development in Streamwise Planes. It is interesting to examine the flow development along the impeller blade passages of baseline compressor and the one with CT-1 casing treatment. Figure 19 shows the locations of streamwise observation planes from impeller inlet to exit. Plane-1 is at inducer inlet, Plane- 3 is at the trailing end of the treatment slot, and Plane-7 is at impeller exit.

Figure 20 shows the relative Mach number distribution on seven streamwise planes (see Figure 19) from inducer inlet to impeller exit for baseline impeller (Figure 20(a)) and for impeller with CT-1 configuration (Figure 20(b)) at operating points corresponding to the stall mass flow rate of baseline impeller. In case of baseline impeller (Figure 20(a)), the flow has normal Mach number distribution at inducer inlet. Downstream of the leading edge, the flow shows retardation at the shroud wall in the convex region from Plan-2 to Plane5. The low momentum recirculating fluid zone extends from impeller suction surface to pressure surface and occupies almost $25 \%$ of the blade height from shroud. In fact, the low Mach number region can be seen along the entire blade height on the suction surface. As the flow moves downstream (Plane-3 and Plane-4), the recirculation zone tends to shift towards pressure surface, and the flow on the suction surface below the recirculation region improves. However, at Plane5 , the recirculation region becomes smaller and shifts again towards suction surface. As the flow proceeds further downstream, the recirculation zone almost disappears, as can be seen at Plane- 6 and Plane-7 (impeller tip). This large scale flow recirculation in the inducer shroud region is responsible for the onset of stall.

The flow behavior within the impeller passage drastically changes in the presence of casing treatment CT-1, as shown in Figure 20(b). The recirculating flow region at and near the inducer disappears (Plane-2 and Plane-3) due to incoming high momentum flow entering straight into the casing treatment slots. However, a small region of recirculating low momentum flow appears at Plane-3 near the pressure surface. It becomes stronger at Plane- 4 and spreads out across the blade pitch in the shroud region as the flow proceeds to Plane-5. Further downstream (Plane-6 and Plane-7), the flow quality improves as was the case with the baseline impeller at its stall mass flow rate (Figure 20(a)). Overall, the improvement in flow quality with casing treatment CT1 is quite remarkable and this allows the mass flow rate to be reduced further with resulting improvement in stall margin.

Even at stall mass flow rate for impeller with CT-1 (Figure 21), the flow quality at Plane-2 is far better than that for baseline impeller (Figure 20(a)). At Plane-3, a relatively small recirculating flow region appears on the suction surface near impeller tip (shroud). However, it spreads across radial and tangential directions and shifts towards pressure surface as the flow proceeds to Plane-5. The behavior is similar to the baseline case at stall mass flow rate, but the strength of the recirculation zone is relatively smaller. At Plane-6, this weak recirculating region splits in two parts that are attached to hub and shroud walls. At impeller exit (Plane-7), the flow becomes more or less uniform. Thus, it can be seen that the overall flow quality through the impeller with casing treatment CT-1 


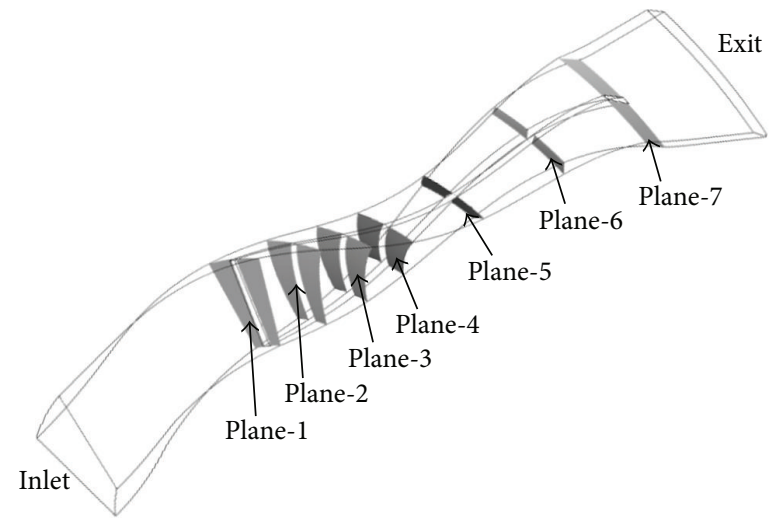

FIGURE 19: Observation planes in streamwise direction from inducer inlet to impeller exit.

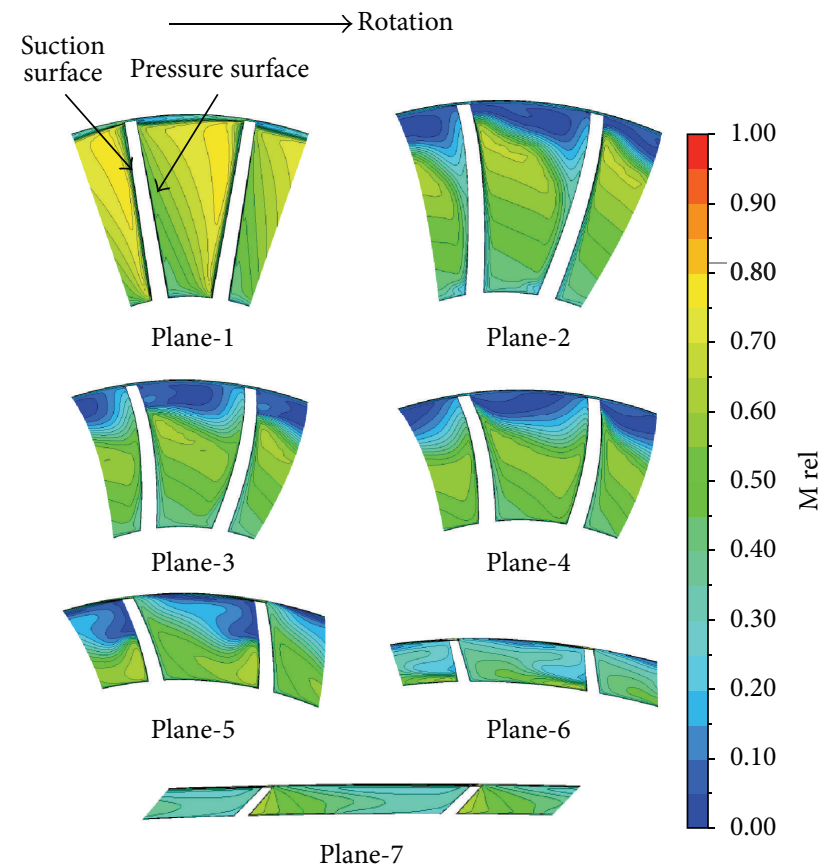

(a) Baseline impeller

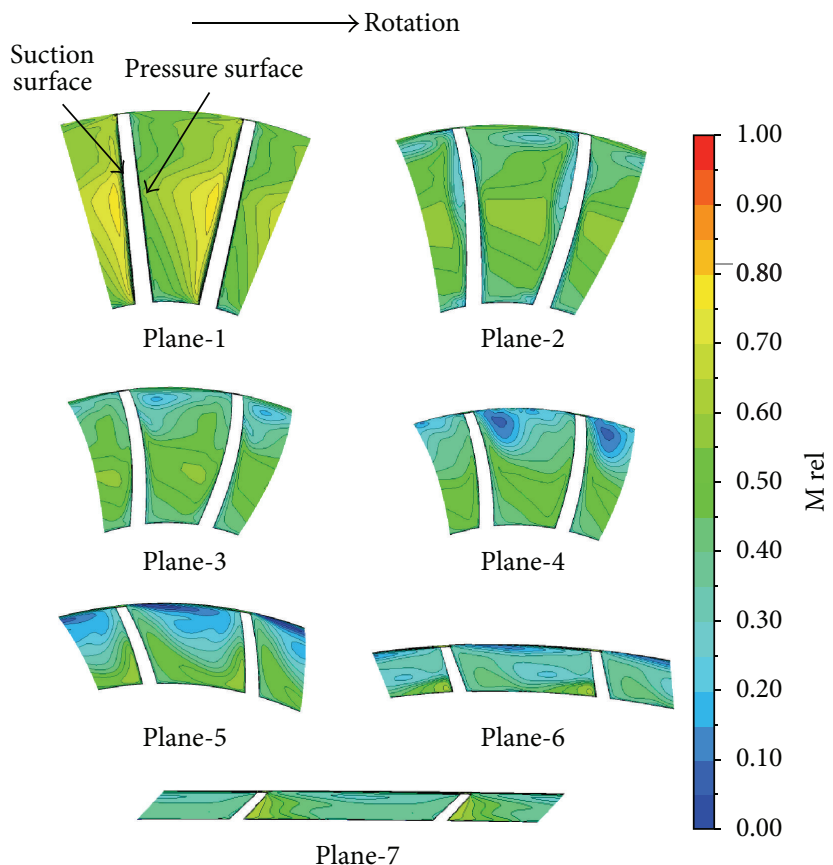

(b) Impeller with CT-1

FIGURE 20: Relative Mach number distribution on streamwise planes of baseline and CT-1 impeller at stall mass flow rate of baseline compressor.

is better than that of the baseline line impeller at respective stall mass flow rates.

4.7. Flow Development in Spanwise Planes. Figures 22 and 23 show the variations of relative Mach number and entropy, respectively, in the impeller blade passage at $90 \%$ span from hub at operating point corresponding to the stall mass flow rate of baseline impeller. Comparing Figures 22(a) and 22(b), the improvement in flow quality brought about by casing treatment in the inducer region of the compressor is clearly seen. Similarly, comparing the entropy distribution in Figures 23(a) and 23(b), it is recognized that the entropy levels in the forward portion of the impeller with casing treatment are much lower than those without casing treatment. This improvement in the flow quality with casing treatment CT1 is mainly due to straight flow entry through the treatment slots.

The effect of inducer casing treatment on the tip leakage flow is depicted in Figure 24. Relative velocity vectors are plotted on a streamwise plane near the impeller tip for baseline and CT-1 impellers at an operating point corresponding to the stall mass flow rate of baseline impeller. In case of impeller without casing treatment (Figure 24(a)), the incoming relative flow is chaotic right from inducer inlet to the impeller exit. The relative velocity inside the inducer passage near the tip is low, as has been discussed earlier, and the flow tends to cross the tip gap right at the inducer. Owing to large incidence angle at low mass flow rate, the flow 


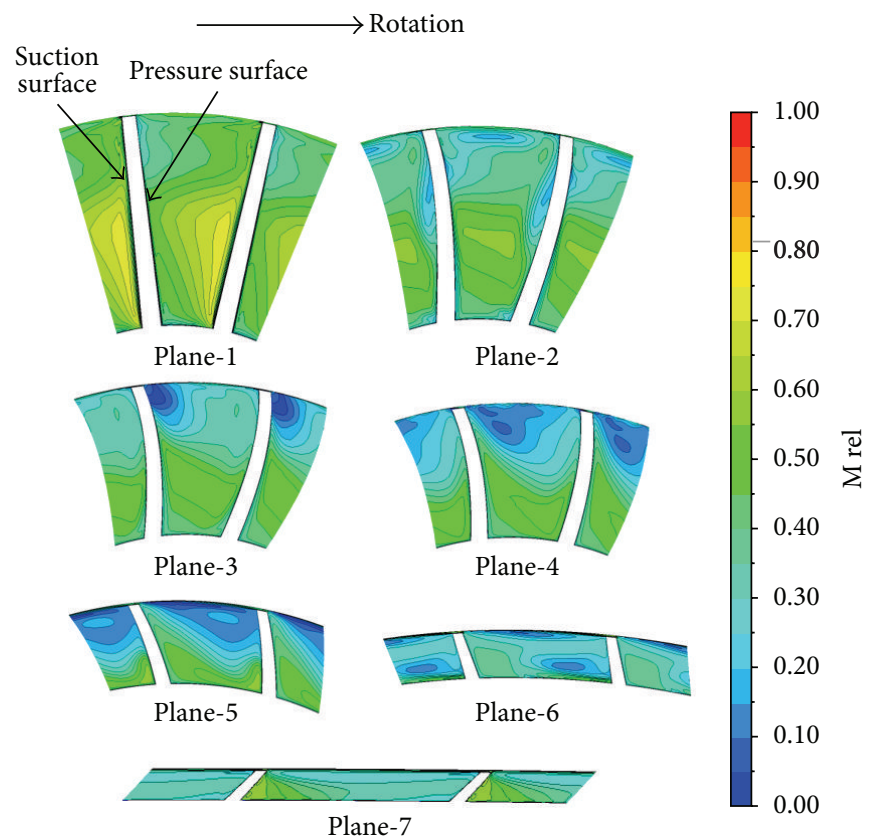

FIGURE 21: Relative Mach number distribution on streamwise planes of CT-1 impeller at stall mass flow rate.

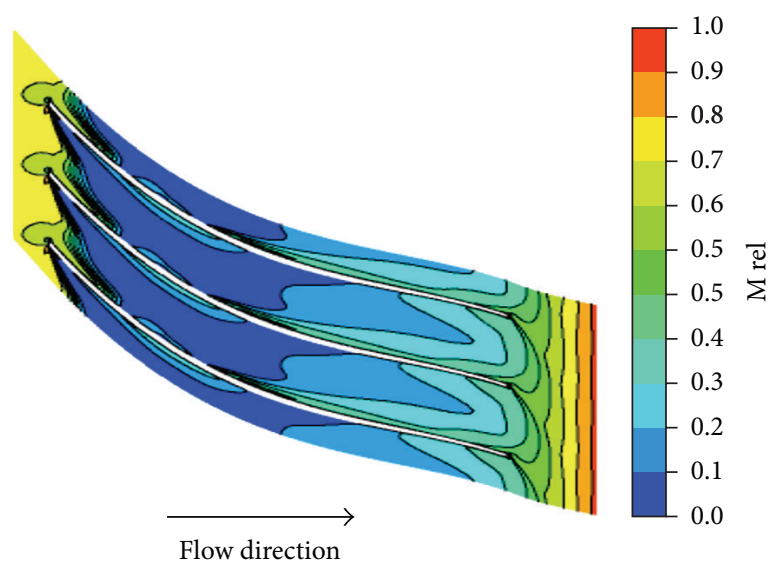

(a) Baseline impeller

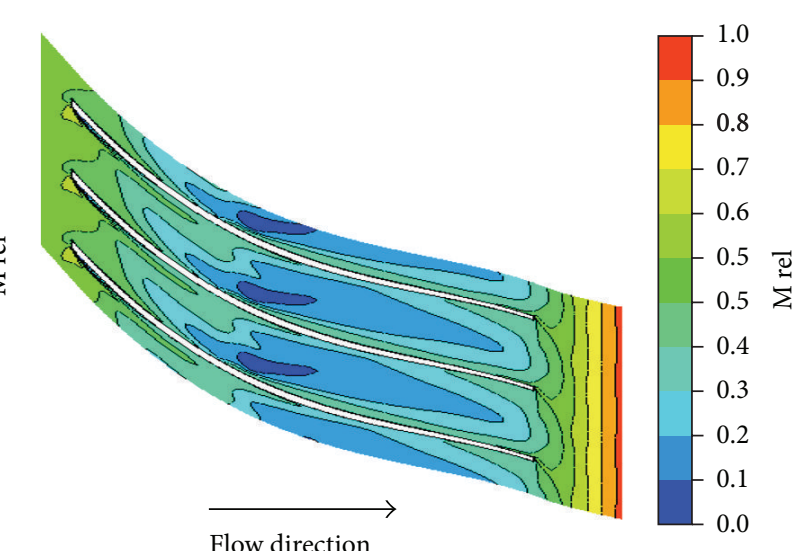

(b) Impeller with CT-1

Figure 22: Relative Mach number distribution on spanwise planes of baseline and CT-1 impellers at $90 \%$ span from hub at stall mass flow rate of baseline compressor.

separates on the suction surface. The resulting partial flow blockage initiates compressor stall. In the presence of casing treatment CT-1 (Figure 24(b)), the flow inside the inducer passage is energized and its chaotic nature disappears. The velocity vectors are aligned to the blade contour with no crossover across the tip gap. Eventually, as the relative flow aligns to the blade angle, the incidence angle reduces with consequent weakening of separated flow region and flow blockage. This allows further lowering of mass flow rate leading to improvement in stall margin. Further downstream, when the pressure builds up, there is tip leakage across the blade surfaces, similar to the baseline impeller.

4.8. Effect of Casing Treatment on Choke Mass Flow Rate. As discussed earlier in Section 4.2 (Figure 12), the choke mass flow rate increases by $9.5 \%$ for all three casing treatment configurations compared to the baseline impeller. It is worth discussing the fluid dynamic reasons behind this enhancement of choke mass flow rate with casing treatment leading to overall increase in the operating range of the compressor. Figure 25 shows circumferentially mass averaged relative Mach number distribution in meridional plane at respective choke mass flow rates for baseline and CT-1 impeller builds. Figure 26 compares the relative Mach number at $90 \%$ span for baseline and CT-1 impellers at respective choke mass flow rates.

Referring to Figures 25(a) and 26(a), it is observed that, under high mass flow rate and low back pressure conditions, the flow accelerates on the suction surface of the inducer of baseline impeller downstream of the leading edge. Eventually, 


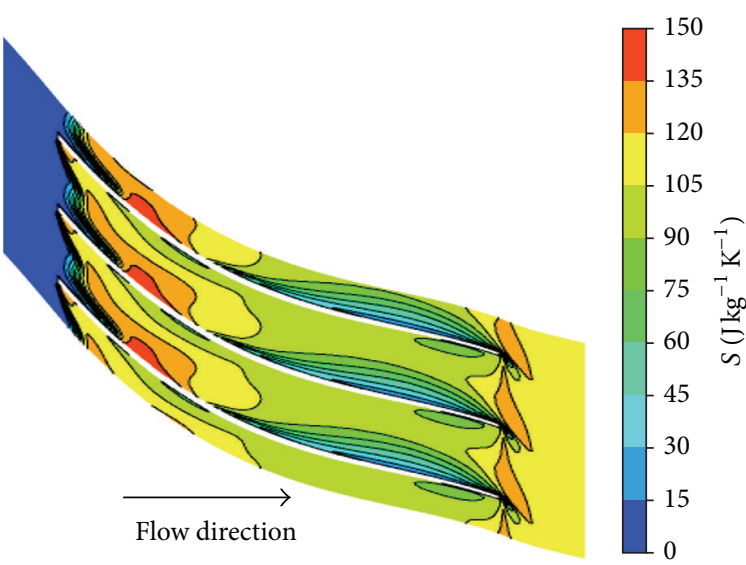

(a) Baseline impeller

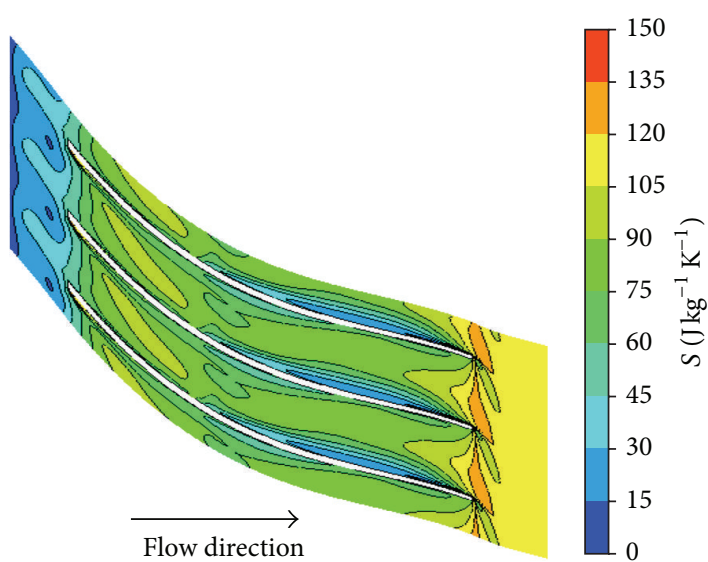

(b) Impeller with CT-1

FIGURE 23: Entropy distribution on spanwise planes of baseline and CT-1 impellers at 90\% span from hub at stall mass flow rate of baseline compressor.

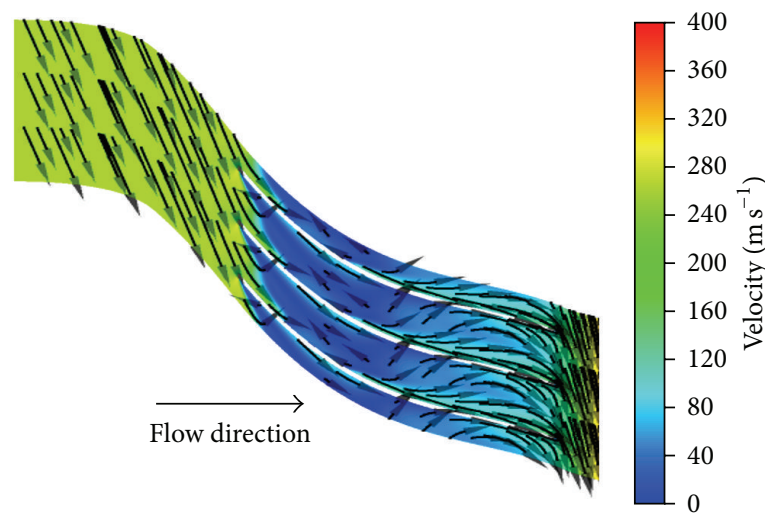

(a) Baseline impeller

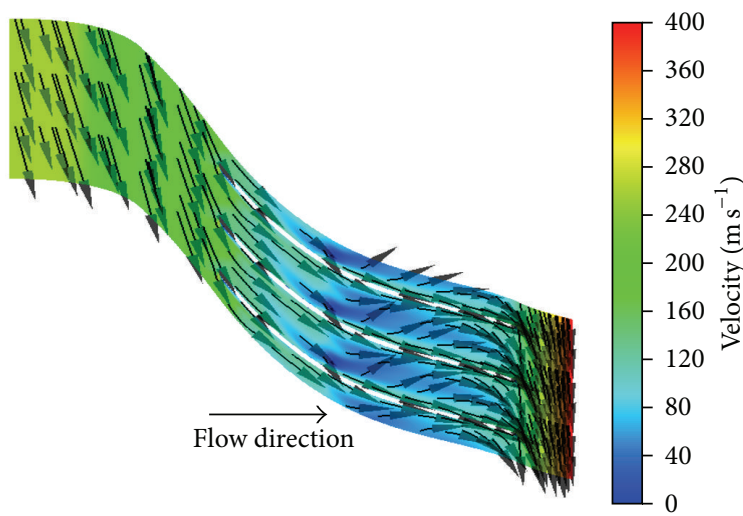

(b) Impeller with CT-1

FIGURE 24: Relative velocity vectors on spanwise planes of baseline and CT-1 impellers near tip at stall mass flow rate of baseline compressor.

there is choking when the flow Mach number reaches unity. Further downstream, the flow decelerates under the influence of adverse pressure gradient up to impeller exit. In case of casing treatment at impeller inlet in the inducer region (Figures 25(b) and 26(b)), the slot openings provide additional area for the flow to pass. Consequently, the mass flow rate tends to increase and the accelerated fluid zone moves downstream of the inducer at the location of convex curvature on the shroud. Again the flow tends to choke when the Mach number approaches unity but at increased mass flow rate. Further downstream, the flow decelerates as in the case of baseline impeller. As shown in Figure 12, all the three casing treatment configurations tend to enhance the choke mass flow rate owing to additional inlet opening provided by the slots. The effect of inducer casing treatment slots at high mass flow rates is more or less similar to an impeller with splitter vanes, wherein the inlet flow area is larger, which tends to allow higher mass flow rate than the impeller with all full length blades.

\section{Conclusions}

Numerical investigations have been carried out on a high speed centrifugal compressor of design pressure ratio $4: 1$ to determine the effectiveness of casing treatment technique in enhancing the compressor aerodynamic performance. Three casing treatment geometries, differing in inlet configuration and respective lengths (upstream of the inducer leading edge), were incorporated in the shroud over the inducer. The treatment slots of identical porosity were designed to follow the profile of the inducer tip section. The CFD simulations were carried out at $100 \%$ design speed from choke to stall mass flow rate. The major conclusions drawn from this study are presented below:

(i) All three casing treatment configurations tend to improve stall margin of the centrifugal compressor with slight reduction in isentropic efficiency. The configuration CT-1 with a larger inlet diameter gives highest stall margin improvement of $18 \%$, whereas 


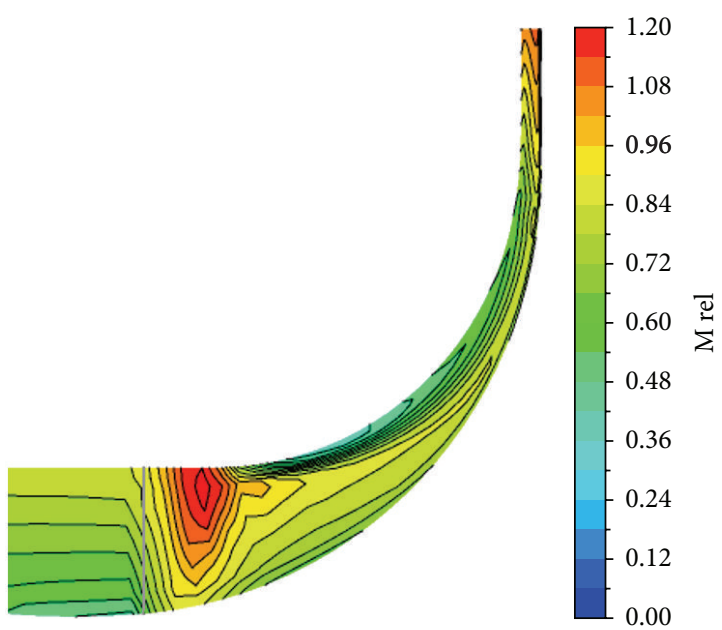

(a) Baseline impeller

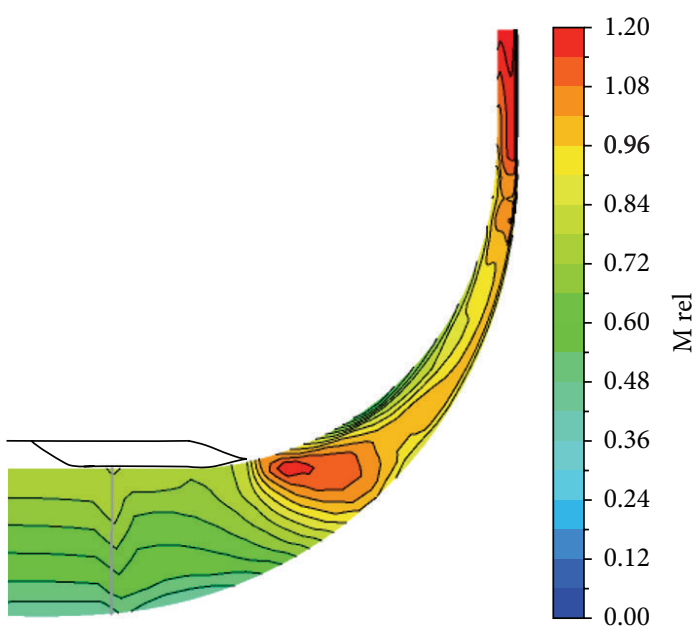

(b) Impeller with CT-1

FIGURE 25: Circumferentially mass averaged relative Mach number distribution in meridional plane at respective choke flow rates of baseline and CT-1 impellers.

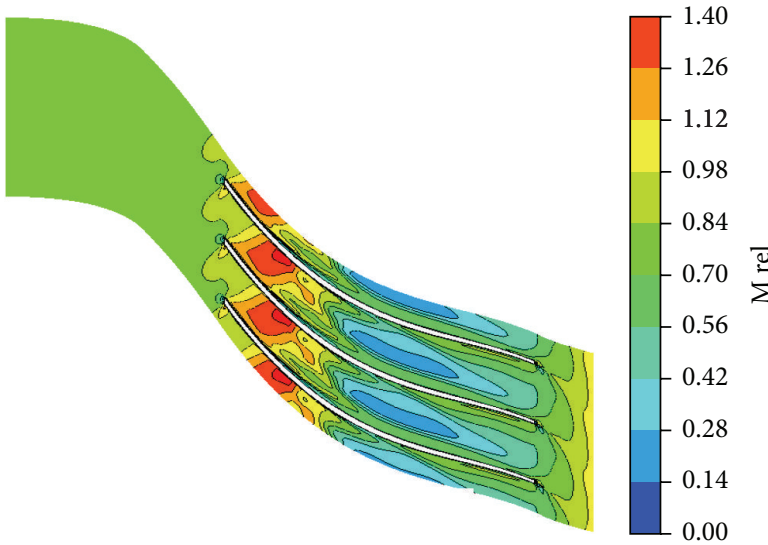

(a) Baseline impeller

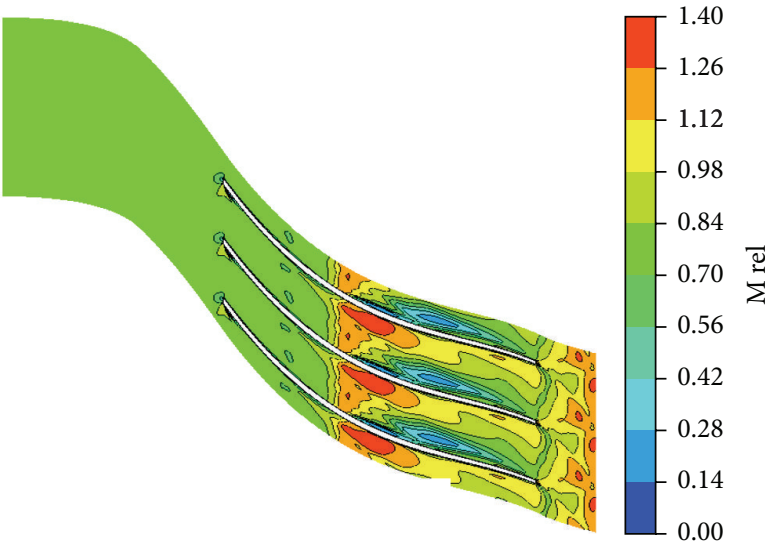

(b) Impeller with CT-1

FIGURE 26: Relative Mach number distribution on a spanwise plane $90 \%$ from hub at respective choke flow rates of baseline and CT-1 impellers.

for the other two configurations CT-2 and CT-3 the stall margin improvement is relatively much smaller. Peak efficiency of the impeller with CT-1 is reduced by $0.8 \%$ and with CT- 2 and CT- 3 by $1 \%$ compared to the baseline impeller.

(ii) The casing treatment CT-1 with its outer diameter aligned with the diameter of the compressor inlet duct is able to push the incoming flow straight through the slots, thus energizing the low momentum fluid at the compressor shroud. The recirculating flow region is weakened and pushed downstream. The resulting reduction in flow blockage is responsible for lowering of stall mass flow rate.

(iii) The casing treatments CT-2 and CT- 3 , on the other hand, have slots whose inner diameter is aligned with the compressor inlet diameter. The flow through the slots is not strong enough to weaken the recirculating flow region of baseline impeller. Also, there is reverse flow in the vicinity of the inducer tip. Hence, these two CT configurations are not effective in reducing the flow blockage and the improvement in stall margin is marginal.

(iv) The choke mass flow rate of impellers with all three casing treatment configurations is higher than that of the baseline impeller. This is attributed to additional flow area through casing treatment slots available at the compressor inlet.

(v) Overall, the stable operating range from choke to stall mass flow rate is improved by all three casing treatment configurations investigated.

\section{Nomenclature}

M: Mach number

$N$ : Speed, rpm 
$P_{s}:$ Static pressure, $\mathrm{Pa}$

$P_{o}$ : Total pressure, $\mathrm{Pa}$

$T_{o}$ : Total temperature, $K$

$m$ : Mass flow rate, $\mathrm{kg} / \mathrm{s}$

$\delta$ : Ratio of compressor inlet total pressure to sea level total pressure

$\gamma$ : Ratio of specific heats

$\eta$ : Isentropic efficiency

$\theta$ : Ratio of compressor inlet total temperature to sea level total temperature.

\section{Suffix}

1, 2: Impeller inlet, impeller exit

abs: Absolute

rel: Relative.

\section{Abbreviations}

CFD: Computational Fluid Dynamics

CAD: Computer Aided Design

CT: Casing treatment

LE: Leading Edge

NASA: National Aeronautics and Space Administration

PS: $\quad$ Pressure surface

SS: $\quad$ Suction surface

TE: Trailing edge.

\section{Disclosure}

V. V. N. K. Satish Koyyalamudi is currently working at GE, Oil \& Gas, Bangalore, India.

\section{Competing Interests}

The authors declare that there are no competing interests regarding the publication of this paper.

\section{Acknowledgments}

The authors are thankful to the authorities of M. S. Ramaiah University of Applied Sciences, Bangalore, India, for providing all support to carry out this research work and for granting permission to publish the same in the form of this paper.

\section{References}

[1] C. C. Koch, "Experimental evaluation of outer casing blowing or bleeding of single stage axial flow compressor," Part VI, Final Report NASA CR-54592, 1970.

[2] W. M. Osborn, G. W. Lewis Jr., and L. J. Heidelberg, "Effect of several porous casing treatments on stall limit and on overall performance of an axial rotor," NASA TND 6537, 1971.
[3] E. E. Bailey and C. H. Voit, "Some observations of effect of porous casing on operating range of a single axial flow compressor rotor," NASA TMX-2120, 1970.

[4] R. D. Moore, G. Kovich, and R. J. Blade, "Effect of casing treatment on overall and blade element performance of a compressor rotor," NASA TND 6538, 1971.

[5] S. A. Guruprasad, Q. H. Nagpurwala, and M. T. Shobhavathy, "Parametric studies on the casing treatment in an axial flow transonic compressor," in Proceedings of the NAL-DLR Workshop on Experimental Fluid Mechanics and Turbomachinery, CSIR-NAL, Bangalore, India, January 2000.

[6] H. Takata and Y. Tsukuda, "Stall margin improvement by casing treatment-its mechanism and effectiveness," Journal of Engineering for Power, vol. 99, no. 1, pp. 121-133, 1977.

[7] G. D. J. Smith and N. A. Cumptsy, "Flow phenomena in compressor casing treatment," Journal of Engineering for Gas Turbines and Power, vol. 106, no. 3, pp. 532-541, 1984.

[8] D. B. Alone, S. Satish Kumar, M. T. Shobhavathy et al., "Performance characterization of the effect of axial positioning of bend skewed casing treatment retrofitted to a transonic axial flow compressor "' in Proceedings of the ASME Turbo Expo: Turbine Technical Conference and Exposition, GT2014-26099, Düsseldorf, Germany, June 2014.

[9] D. B. Alone, S. Satish Kumar, M. T. Shobhavathy et al., "Experimental investigation on the effect of porosity of bend skewed casing treatment on a single stage transonic axial flow compressor," in Proceedings of the ASME Turbo Expo 2014: Turbine Technical Conference and Exposition, GT2014-26102, Düsseldorf, Germany, June 2014.

[10] D. B. Alone, S. S. Kumar, S. Thimmaiah et al., "On understanding the effect of plenum chamber of a bend skewed casing treatment on the performance of a transonic axial flow compressor," in Proceedings of the ASME Turbo Expo 2014: Turbine Technical Conference and Exposition (GT '14), Düsseldorf, Germany, June 2014.

[11] M. P. Boyce, R. N. Schiller, and A. R. Desai, "Study of casing treatment effects in axial compressors," in Proceedings of the ASME Gas Turbine Conference, Zurich, Switzerland, MarchApril 1974.

[12] A. J. Crook, E. M. Greitzer, C. S. Tan, and J. J. Adamczyk, "Numerical simulation of compressor endwall and casing treatment flow phenomena," Journal of Turbomachinery, vol. 115, no. 3, pp. 501-512, 1993.

[13] I. Wilke and H.-P. Kau, "A numerical investigation of the flow mechanisms in a high pressure compressor front stage with axial slots," Journal of Turbomachinery, vol. 126, no. 3, pp. 339349, 2004.

[14] A. Shabbir and J. J. Adamczyk, "Flow mechanism for stall margin improvement due to circumferential casing grooves on axial compressors," Journal of Turbomachinery, vol. 127, no. 4, pp. 708-717, 2005.

[15] R. Emmrich, R. Kunte, H. Hőnen, and R. Niehuis, “Time resolved investigations of an axial compressor with casing treatment-part 2-Simulation," in Proceedings of the ASME Turbo Expo 2007: Power for Land, Sea and Air, Paper No. GT2007-27582, Montreal, Canada, 2007.

[16] W. Jansen, A. Carter, and M. Swarden, "Improvement in surge margin for centrifugal compressors," in Proceedings of the 55th Specialists' Meeting: Centrifugal Compressor, Flow Phenomena and Performance (AGARD '80), AGARD-CP-282, no. 19, Brussels, Belgium, May 1980. 
[17] L. Ding, T. Wang, B. Yang, W. Xu, and C. Gu, "Experimental investigation of the casing treatment effects on steady and transient characteristics in an industrial centrifugal compressor," Experimental Thermal and Fluid Science, vol. 45, pp. 136-145, 2013.

[18] M. T. Barton, M. L. Mansour, J. S. Liu, and D. L. Palmer, "Numerical optimization of a vaned shroud design for increased operability margin in modern centrifugal compressors," Journal of Turbomachinery, vol. 128, no. 4, pp. 627-631, 2006.

[19] P. Gao, Y. Zhang, and S. Zhang, "Numerical investigation of the different casing treatment in a centrifugal compressor," in Proceedings of the Asia-Pacific Conference on Wearable Computing Systems (APWCS '10), pp. 51-54, Shenzhen, China, April 2010.

[20] V. C. Arunachalam, Numerical studies on the effect of impeller blade skew on centrifugal compressor performance [M.S. thesis], M. S. Ramaiah School of Advanced Studies, Bangalore, India, 2008. 


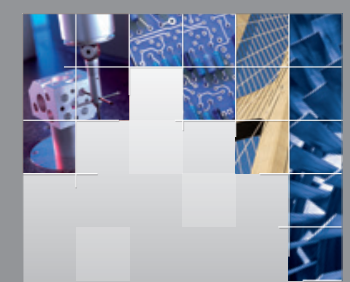

\section{Enfincering}
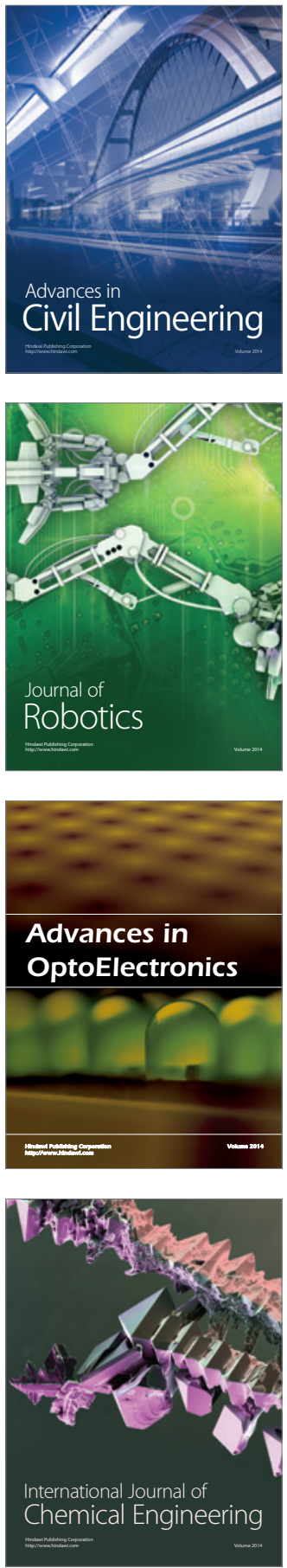

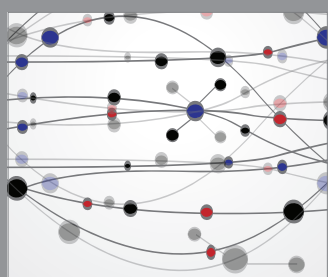

The Scientific World Journal

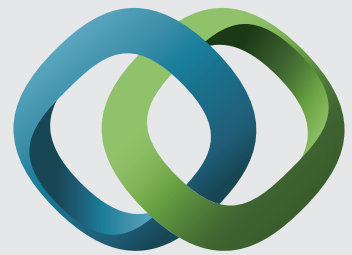

\section{Hindawi}

Submit your manuscripts at

http://www.hindawi.com
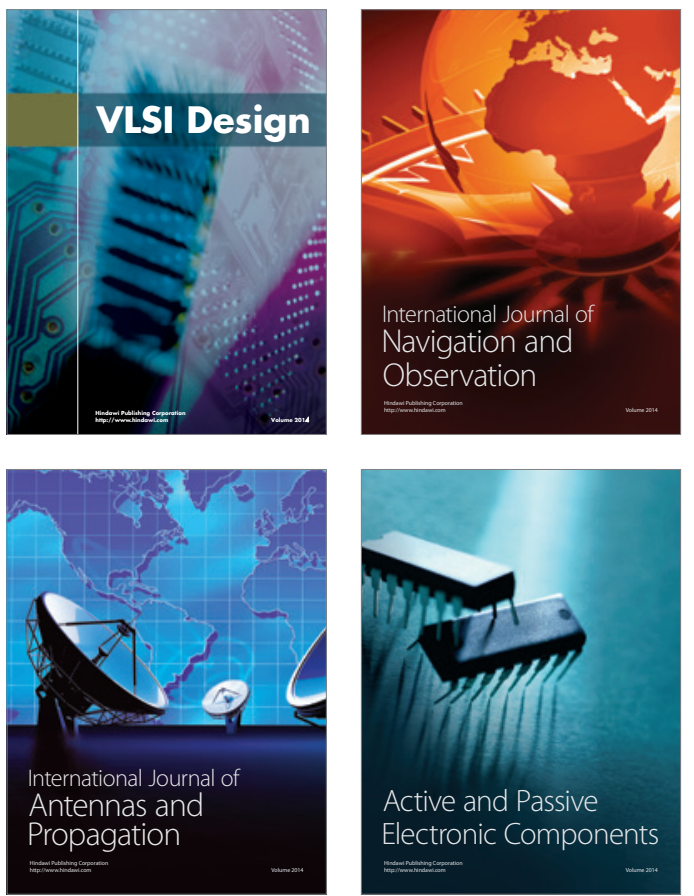
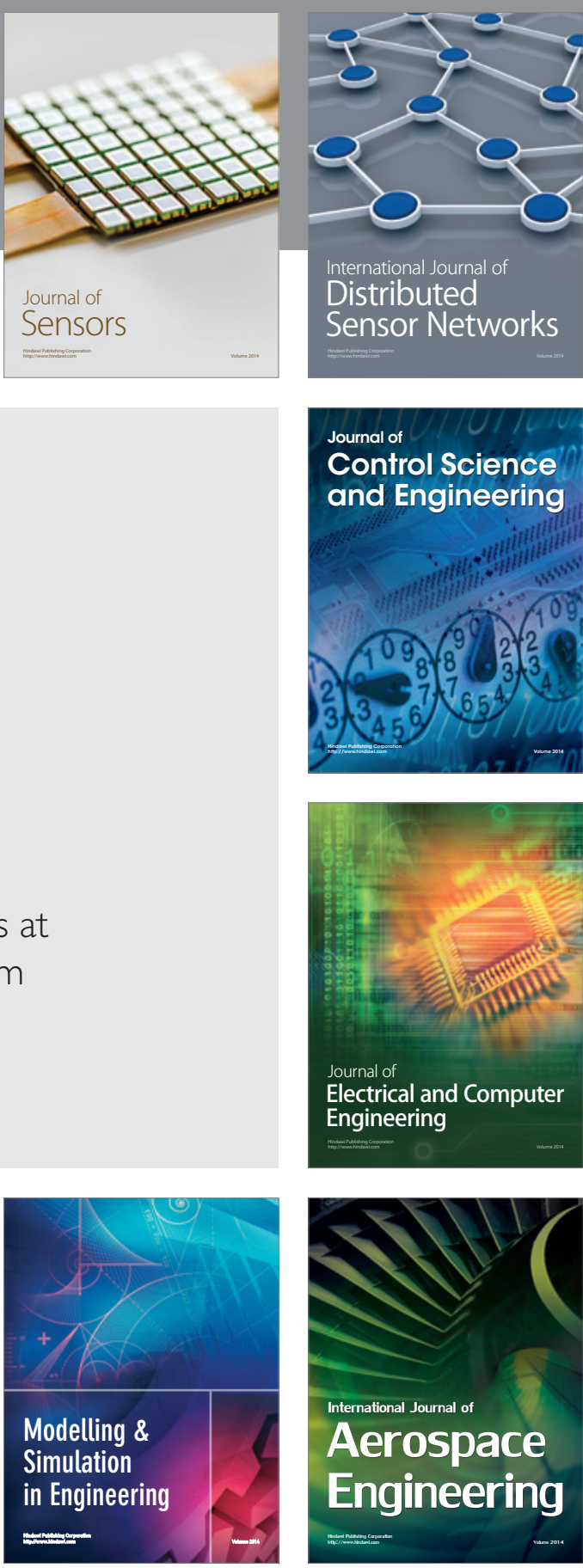

International Journal of

Distributed

Sensor Networks

Journal of

Control Science

and Engineering
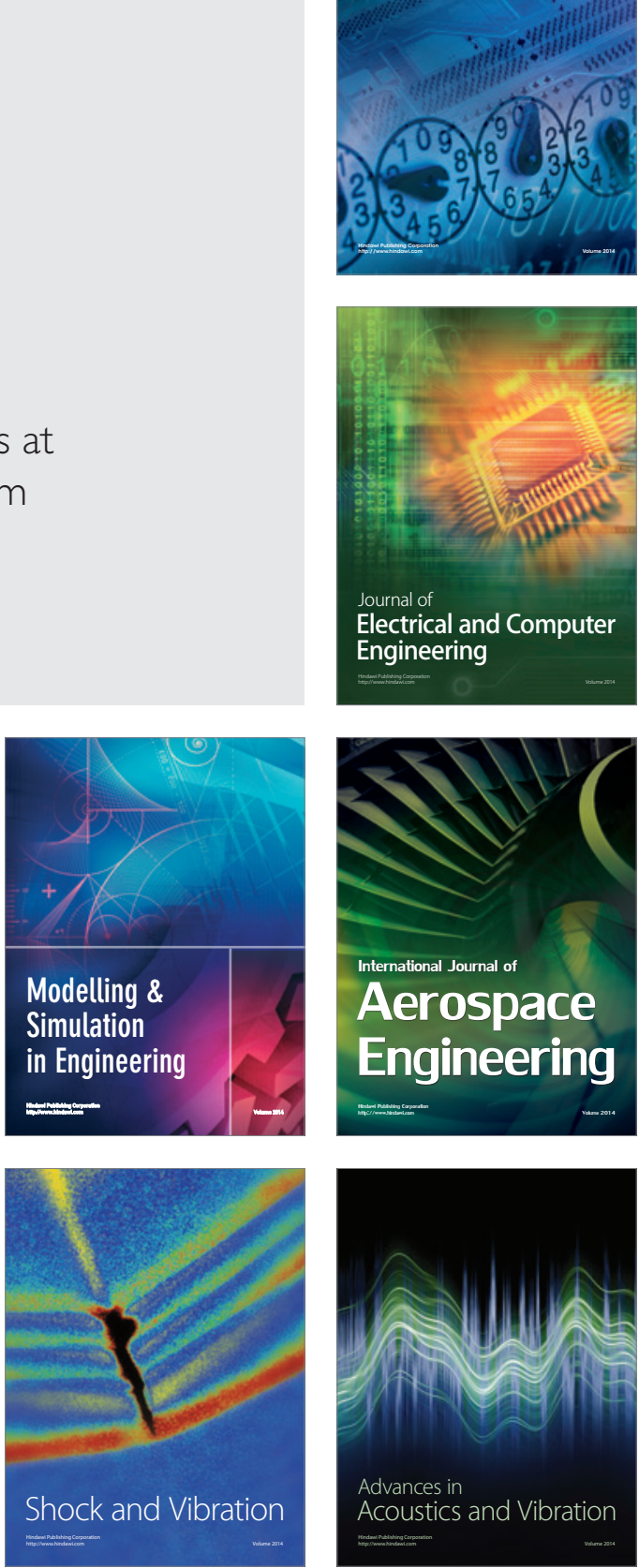